\title{
Towards Next Generation "Smart" Tandem Catalysts with Sandwiched Mussel-inspired Layer Switch
}

\author{
Wenjing Wei ${ }^{1,2}$, Vijay Kumar Thakur ${ }^{1,2,3}$ *, Y. M. John Chew ${ }^{4}$, Songjun $\mathrm{Li}^{2 *}$ \\ ${ }^{1}$ Enhanced Composites and Structures Center, School of Aerospace, Transport and \\ Manufacturing, Cranfield University, Bedfordshire MK43 0AL, UK; \\ ${ }^{2}$ Research School of Polymeric Materials, School of Materials Science \& Engineering, \\ Jiangsu University, Zhenjiang, China \\ ${ }^{3}$ Biorefinng and Advanced Materials Research Center, Scotland's Rural College \\ (SRUC), Kings Buildings, Edinburgh, EH9 3JG, UK \\ ${ }^{4}$ Centre for Advanced Separations Engineering, Department of Chemical Engineering, \\ University of Bath, Claverton Down, Bath BA2 7AY, U.K.
}

\begin{abstract}
In this paper, we prepared a novel reactor with switchable ability to address present challenges in tandem catalyst. By introducing mussel-inspired moiety, this goal was achieved via preparing a "smart" polymer reactor which can open or closes the entry tunnel of the targeted substrate in cascade reactions. The catalyst consisted of two functional layers acting as tandem catalytic parts and one smart layer with musselinspired moieties as a controlled middle switch. The top and the bottom layer were made of molecularly imprinted polymers and catalytic components, like acidic parts and metal nanoparticles, respectively. The middle layer made of polymeric dopamine (PDPA) and acrylamide with self-healing ability will allow or inhibit the intermediate product for the reaction, thus controlling the process of the tandem catalysis. As a result, the novel catalyst exhibited self-controlled tandem catalysis, which provides new opportunities to design smart tandem catalysts, showing a promising prospect in this area.
\end{abstract}

\footnotetext{
* Email address: Vijay.Kumar@cranfield.ac.uk (V.K. Thakur)

* Email address: 1sjchem@ujs.edu.cn (S.Li)
} 
Keywords: Smart polymers; tandem catalysis; self-controlled switch.

\section{Introduction}

Tandem catalysis is of great significance in the modern chemical industry for cutting down preparation, post-treatment processes, as well as reducing the amount of commonly used solvents $[1,2]$. The use of tandem catalysts would enable complicated multi-steps reactions to be conducted in sequence efficiently, thus resulting in one-pot self-switchable property $[3,4]$. Catalytic reactors with both acid and alkaline sites are among the earliest trials to achieve tandem processes $[5,6]$. In the reactors, the acidic and basic sites are respectively responsible for two different reactions, giving access to the tandem catalysis. One of the remaining challenges in tandem catalysis is that a large number of active intermediates and initial reactions mixed in the system results in a series of unexpected reactions, which has limited the development of the tandem catalysis [7, 8]. Hence, it is important to achieve the precise control and sensible separation of several different catalytic sites to prevent the unnecessary steps in the process.

Human beings have acquired a lot of inspirations and experience from nature to enable us to solve many complicated problems. One exquisite solution of these inspirations is using molecularly imprinted polymers (MIPs) to achieve molecular recognition and selective catalysis [9]. MIPs attracted significant interests of researchers because of their special abilities such as facile preparation and special recognition [10]. To prepare MIPs, firstly, functional monomers are assembled with template molecules to form a complex by covalent or non-covalent bonds. The functional monomers can polymerize in the presence of initiators and cross-linkers under appropriate photo or thermal conditions to form MIPs. The imprinted moieties are then removed from the polymer system under firm conditions leaving behind a cavity that is harmonizing in shape and size to the template. The residual cavity can, therefore, selectively bind to the desired molecules $[11,12]$. In this way, the imprinted catalysis allows the target reaction and avoid the redundant side effects, achieving desired tandem catalysis. However, it is also necessary to control the tandem catalysis process as there are many different kinds of reactions and specific needs in practical 
use [13]. As a result, the tandem catalysts need further improvement to prepare a more delicate structure to perform intelligent tandem catalysis.

Marine organisms such mussels exhibit prominent adhesive ability and is a promising prospect of smart polymer reactors. Marine mussels are capable of affixing themselves to almost all solid substances, containing rocks, metals and even polytetrafluoroethylene (PTFE) [14, 15]. It is worth noting that the strong adhesive property of mussels takes place over an extensive range of weathering and environmental conditions such as temperature, salinities and water currents [16]. So far molecular mechanism involved in this reaction is poorly understood, while clues to the strong adhesive exist in the components of the amino acids that have a lot of lysine and 3,4-dihydroxyphenylalanine (DOPA) segments [17, 18]. It has been reported that hydrogen bonding, metal chelation or radical-surface coupling from the catechol moiety in DOPA are critical to the adhesion $[19,20]$. Based on the strong interactions, mussel adhesive holdfasts also demonstrated distinct self-healing capabilities. The damage sites at dopamine polymers will heal itself totally after being cut, which results from the strong interaction between amino parts and dopamine [21]. This self-assembly ability in the aqueous surrounding of mussel's adhesive proteins provides a new solution to the self-controlled catalysis, giving access to the switchable polymer reactors in an aqueous environment.

Inspired by the exquisite structure in nature, herein, we prepared a tandem catalyst with a sandwiched switch to achieve the controlled tandem catalysis. By introducing the dopamine moiety into the tandem catalyst, the prepared reactor was capable of acting as a switch to "open" and "close" to control the catalytic reactions intelligently. Moreover, to avoid the undesired side reactions in the tandem process, the catalyst was fabricated with two unique imprinted layers to recognize the substrate distinctively. As outlined in Scheme 1, the top and bottom layers were respectively composed of initialimprinted and intermediate-imprinted polymer hydrogel to perform the tandem catalysis. In detail, the top layer was prepared by the acidic monomer while the bottom layer consisted of the functional monomer containing metal nanoparticles, which was able to accomplish the catalytic hydrolysis and reduction process. Besides, 4- 
nitrophenyl acetate (NPA) along with 4-nitrophenol (NP) were respectively chosen as the experimental initiate reactant and intermediate because these two are well-coupled for tandem testing. Meanwhile, the hydrolytic and reductive processes of NPA and NP could be conducted in normal conditions e.g. room temperature [22]. The middle layer, performing as switch between the top and bottom layers, was composed of polymeric dopamine (PDPA) and polyacrylamide (PAm), which was capable of "opening" and "closing" by the self-healing and dissociating interactions between the two polymers. There are well-studied descriptions available regarding the self-healing mechanism driven by hydrogen bonding [23-25]. In essence, the self-healing behavior driven by hydrogen bonding is the switching of hydrogen bonding. When the temperature is comparatively low, the interactions between hydrogen bonds lead to closed access which inhibits access to contacting the reductive metal nanoparticles sites. However, with the temperature rising, the separation of interactions between hydrogen bonds results in open access which allowed entrance to reach the reductive metal nanoparticles. Hence, the self-healing behavior driven by hydrogen bonding demonstrates the switching behavior.

As clarified in Scheme 1, the inhibited entrance in the middle layer at comparatively low temperatures could prevent the access to the reductive sites of the intermediate from the top layer, resulting in the first catalytic process merely. However, the entrance to the intermediated is open with the rising temperature, enabling further access to reduce the forming intermediate NP and achieving the complete tandem catalysis. Furthermore, the outstanding adhesive ability of dopamine moiety in the middle layer enabled tight junction between different layers even after repeated catalysis processes. As a result, the reactor performed the switchable cascade catalysis. In this study, we try to prepare a novel catalyst which can solve the problems in "smart" multi-step catalysis, suggesting new protocol in the self-switchable tandem catalysis. 


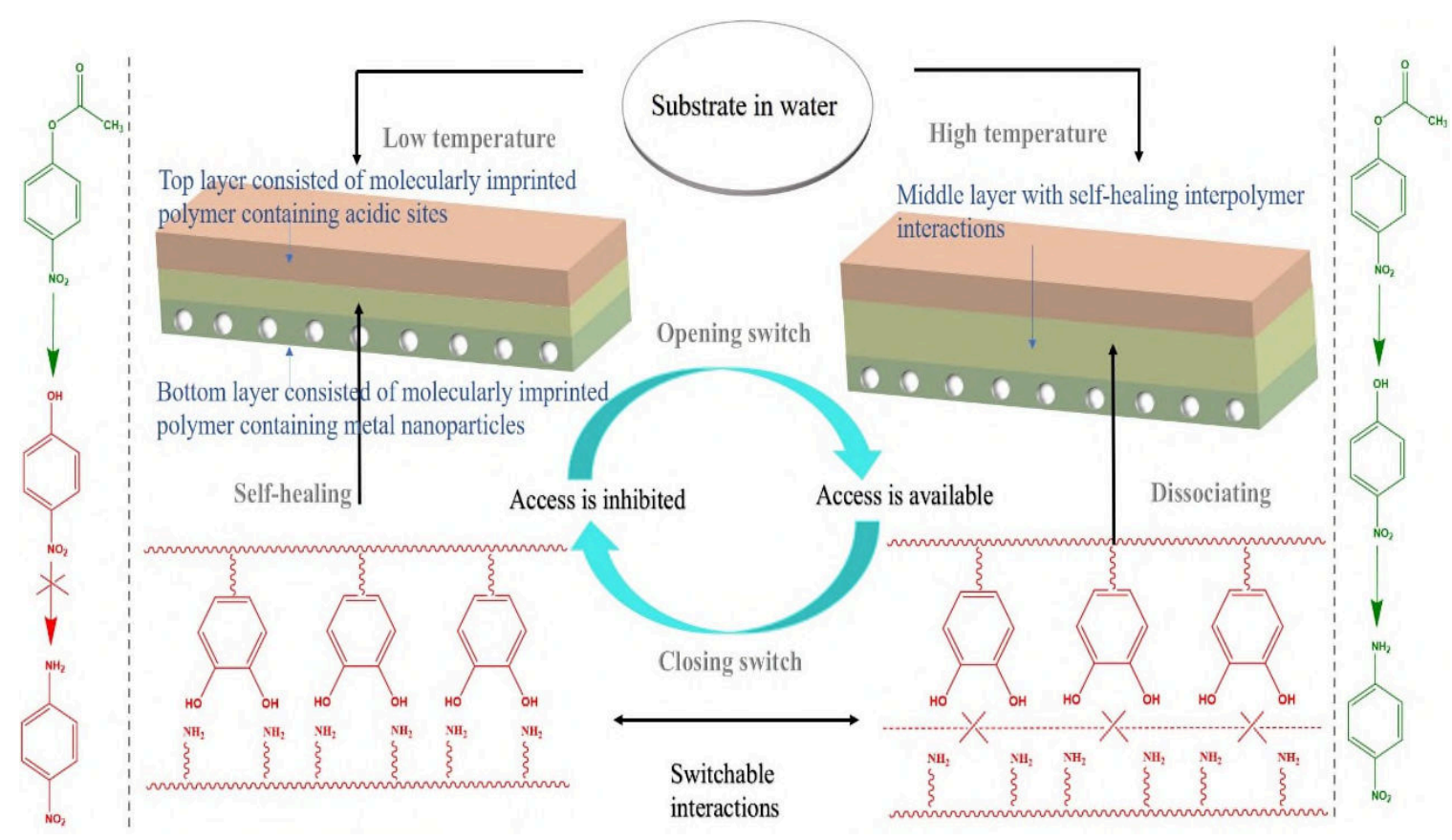

Scheme 1. Self-controlled mechanism of the novel polymer reactor

\section{Experimental Section}

\subsection{Synthesis of polymer reactors}

All the chemicals (analytical grade) in this experiment were bought from SigmaAldrich and were used without further processing. The polymer reactor was synthesized in a three-layer structure consisted of two imprinted cascade layers (top, acidic layer; and bottom, metal encapsulated layer) and a middle switchable layer (PDPA-PAm) sandwiched between the two layers (Scheme 2). In the preparation process, firstly, part of the top imprinted layer was immersed in the middle layer solution after synthesizing the top layer completely [26]. And there will be an interpenetrated network in the joint part between layers after the polymerization of the middle layer because of the solution diffusing to the top layer. The bottom layer was also polymerized by the same process. Therefore, all three layers were linked together.

To fabricate the three-layer reactor, 2-acrylamido-2-methylpropane sulfonic acid (AMPS) (2.33 g), azobisisobutyronitrile (AIBN) (0.09 g), N, N' -methylene bisacrylamide (MBA) (0.35 g) and imprinted substrate 4-nitrophenyl acetate (NPA) (0.407 g) were dispersed completely in dimethyl sulfoxide (DMSO) (10 mL). The mixed solution was dissolved by ultrasonic machine and then deoxygenated totally, 
then it was put in a vacuum at $70{ }^{\circ} \mathrm{C}$ for 3 hours to polymerize the top imprinted layer. Afterwards, part of the top imprinted layer was immersed in the middle layer solution. This mixed solution was composed of dopamine acrylamide (DPA, nomenclatural known as N- [2-(3, 4-dihydroxy phenyl) ethyl]-2-methylacrylamide, 0.5 g), acrylamide (Am, $1.59 \mathrm{~g})$, divinylbenzene $(0.11 \mathrm{~g})$ and AIBN (0.11 g) in dimethylformamide solution $(10 \mathrm{~mL})$. The synthesis process was conducted at $70{ }^{\circ} \mathrm{C}$ for 5 hours after deoxygenation. At next step, the first two layers were contacted with the bottom imprinted layer solution containing Am (1.6 g), the complex template $\left[\mathrm{Ag}(\mathrm{NP})_{2}\right]^{+}(2.25$ mmol), MBA (0.34 g) and AIBN (0.18 g) mixed components in dimethyl sulfoxide (10 $\mathrm{mL}$ ), performing at $70{ }^{\circ} \mathrm{C}$ for $2 \mathrm{~h}$ to polymerize completely. And the encapsulated $\mathrm{Ag}$ ions were reduced in an excessive amount of sodium borohydride $\left(\mathrm{NaBH}_{4}\right)$ (ten-fold to Ag ions) solution. After reduction, excess deionized water was used to remove the left $\mathrm{NaBH}_{4}$. Then the imprinted parts NPA and NP were washed profusely from the catalytic reactors with ethanol including $10 \%$ acetic acid. As a result, this three-layer polymer reactor was synthesized (called "MIP-AgPRS"; here, "MIP" meant the imprinted polymer, "Ag" represented Ag nanoparticles and "S" stood for switchable properties).

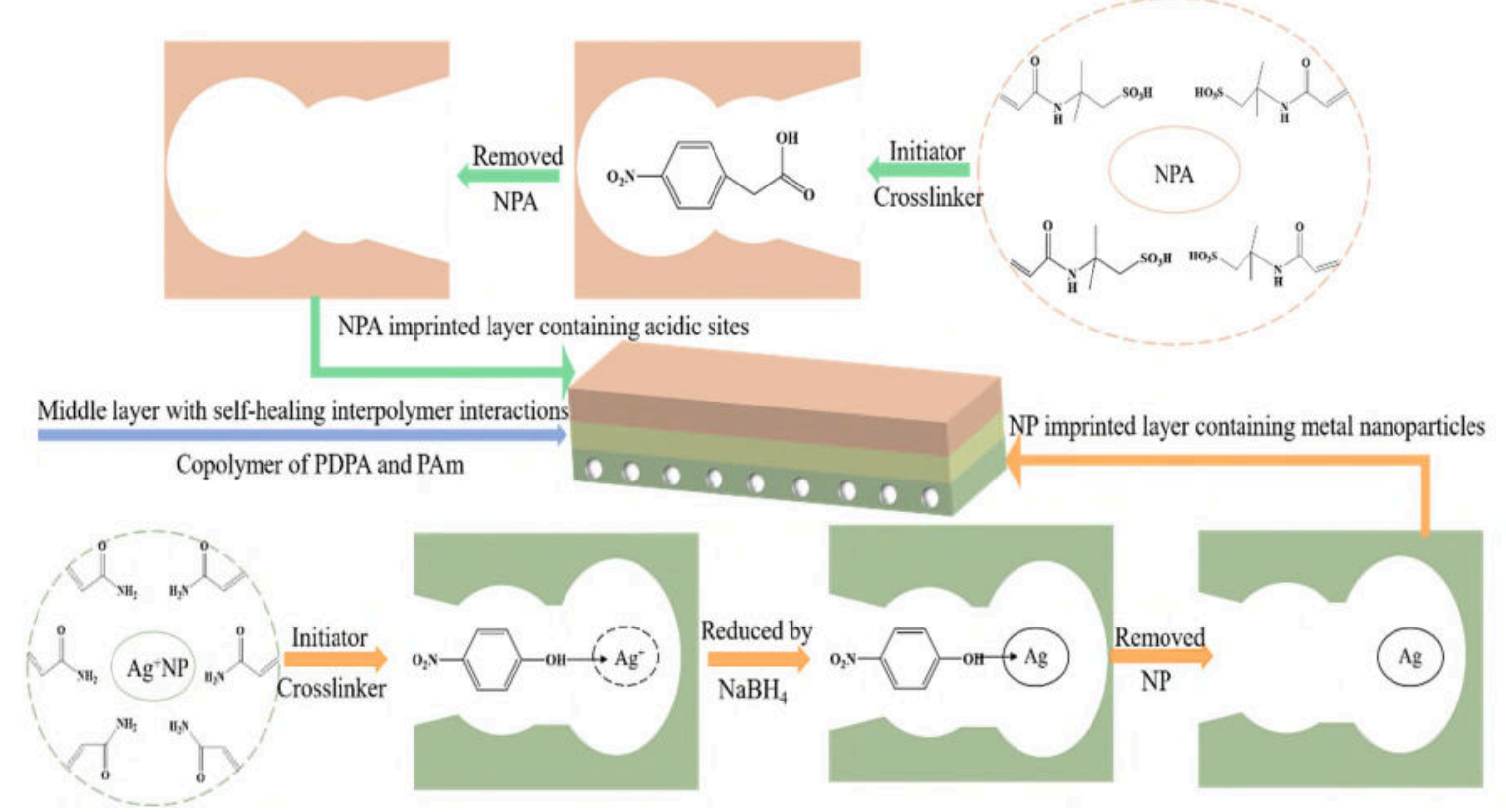

Scheme 2. The preparation of the tri-layer polymer reactor.

To further study the switchable properties of the catalyst MIP-AgPRS in the middle layer and, two other controls respectively name "MIP-AgPRO" and "MIP- 
AgPRC”, were synthesized in similar environments. In comparison with the switchable polymer reactor, MIP-AgPRO also had the acidic and metal contained imprinted layers but the middle layer acted as a switch keeping open ("O"), which was prepared by the polymerization of the Am alone. Besides, MIP-AgPRC was equipped with the middle layer in closed access ("C") all the time, prepared by the PDPA alone. As a result, the one control (MIP-AgPRO) will allow the tandem process at both low and high temperatures, and the other control (MIP-AgPRC) will give access to only the first reaction at a different temperature, which was conceptually comparable to the open or closed statuses with the prepared reactor MIP-AgPRS at different temperature. In this way, the prepared catalyst and the controls were prepared.

\subsection{Characterization}

The morphology of the nanoreactor and the dispersion of the Ag nanoparticles were observed by Transmission Electron Microscope (TEM) imaging (Japan; JEM2100). And Fourier Transform Infrared Spectrometer (FTIR) data were collected to study the specific functional groups (USA; Nicolet $M X-1 E)$. The surface plasma resonance (SPR) was used to study the absorption bands of metal nanoparticles (USA; Lambda 25 UV spectrophotometer) and energy-dispersive spectra (EDS) were collected to compare the different element components (USA; MIRA3-XMU) of reactors. A Smartlab-3KW diffractometer (Japan; Rigaku) with $\mathrm{Cu} \mathrm{K} \alpha$ radiation $(\lambda=1.5418 \AA$ ) was used to study X-ray diffraction (XRD) spectra.

\subsection{Self-healing interactions}

The self-healing processes in different temperatures of the switchable layer was recorded by dynamic light scattering (DLS) (UK; Nano ZS90). The products taken from the middle layer were firstly dispersed in water at a specific temperature for $10 \mathrm{~min}$ to reach equilibrium condition before starting to collect the dynamic radius $\left(R_{d}\right)$. Compared with the other two controls without self-healing ability, the switchable interaction between the PDPA and PAm was presented as the changing dynamic radius according to different temperatures. 


\subsection{Temperature-programmed desorption}

Temperature-programmed desorption (TPD) was conducted to investigate the interaction among the substrates and the imprinted layers to clarify the molecular recognition abilities in the top and bottom layers. The sample of recognition properties will show greater retention in comparison with other control reactors without the molecular part. The products $(20 \mathrm{mg})$ were taken from the imprinted layer and were put into a TPD device (in a U-shaped quartz tube). After absorbing with $20 \mu \mathrm{L}$ reactant $\left(0.05 \mu \mathrm{mol} \mathrm{mL} \mathrm{L}^{-1}\right.$ acetonitrile), the products were heated in flowing nitrogen $\left(60 \mathrm{~mL} \mathrm{~min}{ }^{-}\right.$ $\left.{ }^{1} ; 0.45 \mathrm{MPa}\right)$ at the speed of $10{ }^{\circ} \mathrm{C} \mathrm{min}^{-1}$ rising to the desorbing temperature of corresponding reactants from room temperature. The desorbing spectra were collected and analyzed by the data processing system.

\subsection{Catalysis properties}

The catalysis processes of the different polymer reactors were studied using batch system [27]. The starting concentration of NPA was $0.05 \mu \mathrm{mol} \mathrm{mL} \mathrm{mL}^{-1}(10 \mathrm{~mL}$ PBS, pH 7.0). The concentration of catalysts in every test was $0.1 \mathrm{mg} \mathrm{mL}^{-1}$. The catalysis process was spectrophotometrically recorded against time (Japan; $U V-2700$ ) and the final conversion efficiency was decided by the average value of three tests. Due to the existing influence of self-hydrolysis on the catalysis reactions, the hydrolysis of NPA was also conducted on the same environment without the polymer reactors and then was deducted from the whole system. Finally, the catalytic performance of the reactor was presented without the effects of the self-hydrolysis. And the reduction process was further performed followed by the hydrolysis, at the same time adding excessive sodium borohydride (fourfold, relative to NPA) to the system.

\subsection{Electrochemical tests}

The self-healing mechanism (catalytic) among reactors and the reactants were further studied by electrochemical tests. Herein, an electrochemical workstation (China; CHI760E) assembled with a three-electrode cell was used. The prepared reactors (10 mg) absorbed $\sim 2 \mu \mathrm{mol}$ templates in ahead were put in the electrochemical cells and 
then the experiments were carried out in an ultrasonic apparatus to conduct mass diffusion (electrolyte: 10mL PBS; pH 7.0). The desorbing performance in the catalytic reactor was recorded through circularly scanning the system till steady desorption profiles were achieved (scanning range, $0 \sim-1 \mathrm{~V}$; scanning rate, $1 \mathrm{mV} \mathrm{s}^{-1}$ ).

\section{Results and Discussion}

\subsection{Analysis of composition and structure}

The catalyst was synthesized in a three-layer structure consisting of the bilayer molecular imprinting tandem catalyst and a smart switch sandwiched between the bilayer catalyst with "on" and "off" ability. The middle switchable layer was made of PDPA and PAm, the top and the bottom layer was respectively made of PAMPS and PAm with silver nanoparticles. Herein, FTIR test was conducted to determine the components of polymer in all three layers. In Figure 1a, four main bands (2900-3700, $1600-1800,1000-1200$ and $1000-1400 \mathrm{~cm}^{-1}$ ) showed in the spectrum of the three reactors, which were because of several different components in this catalyst, respectively related to $\mathrm{O}-\mathrm{H} / \mathrm{N}-\mathrm{H}, \mathrm{C}=\mathrm{O}, \mathrm{S}=\mathrm{O}$, and $\mathrm{C}-\mathrm{N} / \mathrm{C}-\mathrm{C}$ bonds of the stretching vibration of $[28,29]$. Moreover, the three samples exhibited similar spectra as all the prepared reactors contained the same basic functional groups, which is also shown in Figure 3a that all the three reactors contain the same basic elements. As a result, further investigation of the different components in the three layers was carried out through FTIR analysis. Figure 1b showed the spectra of three layers of the MIP-AgPRS respectively. The presence of the $\mathrm{S}=\mathrm{O}$ bond $\left(\sim 1237\right.$ and $\left.1050 \mathrm{~cm}^{-1}\right)$ along with the other major bands indicated the components in top imprinted layer, consisted of PAMPS, distinguished from the other two layers. It is further indicated from the EDS spectra of Figure $3 \mathbf{b}$ that each of the three layers possessed different elements composition, ascertaining the presence of the three-layer structure. The results indicated the new polymer reactor MIP-AgPRS was a complex composite containing the basic functional groups and elements as expected.

To analyze the imprinting process in NPA and NP imprinted layer, we have also compared the FTIR results of both the two different imprinted layers, the precursor (the 
initial polymer reactor without washing the imprinted moieties) and imprinted substrates NPA and NP, respectively (Figure 1c and Figure 1d). The top NPA layer precursor exhibited the major bands of both top layer MIP-NPA and the substrate NPA in the result. After washing the imprinted substrate NPA from the original polymer reactor (the precursor), the spectrum became comparable to the middle layer (the layer with the same basic functional groups as the AM). Furthermore, the bottom NP layer precursor also demonstrated the main bands of both MIP-NP as well as the substrate NP in the spectrum and resulted in the absence of the major bands of NP after removing the imprinted part. Combined with the synthesis route (Scheme 2), the test suggested the existence of imprinting processes (more analysis and characterization about imprinted process is discussed in Section 3.2).

As is presented in Figure 2, the TEM pictures exhibited morphology of Ag nanoparticles existed in the polymer reactors. The size of Ag nanoparticles dispersed in the reactors is about 7 10 $\mathrm{nm}$. And the existence of Ag nanoparticles was ascertained by the tests of EDS (Figure 3), SPR (Figure 4) (typically at 410nm) [30]. Herein, the content of Ag achieves in the NP imprinted layers were $\sim 11.23$ wt \%. XRD spectra in Figure 5 further confirms the presence of Ag nanoparticles, in which there are obvious Ag diffraction peaks. Figure 6 exhibited the digital pictures of the reactor MIP-AgPRS and two controls. As is shown, this polymer reactor presented in a three-layer structure as expected. In this way, the structure of the catalyst was synthesized as expected.

BET sorption isotherms of different layers of the reactors are shown in Figure 7. It can be seen that the first layer MIP-NPA and the third layer MIP-NP-Ag demonstrated the different microspore distributions $(<10 \mathrm{~nm})$ [31] while there is no distinct distribution of the non-imprinted middle layer. Meanwhile, as shown in Table 1, compared with the middle layer, both of the other two imprinted layers exhibited larger surface areas and higher pore volume, which resulted from the existence of the templates in the synthesis process. Therefore, the imprinted moieties of the polymer reactor are confirmed. 

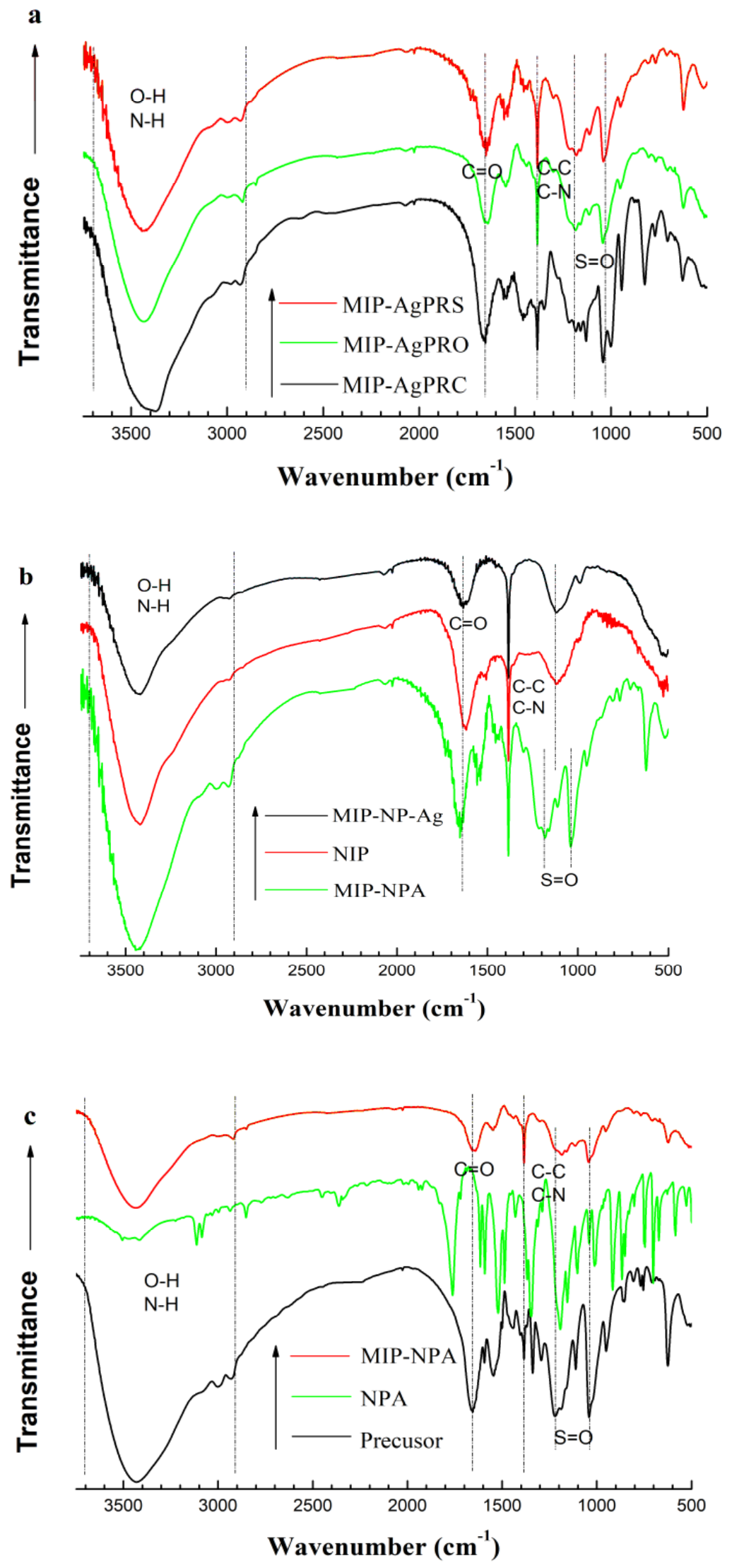


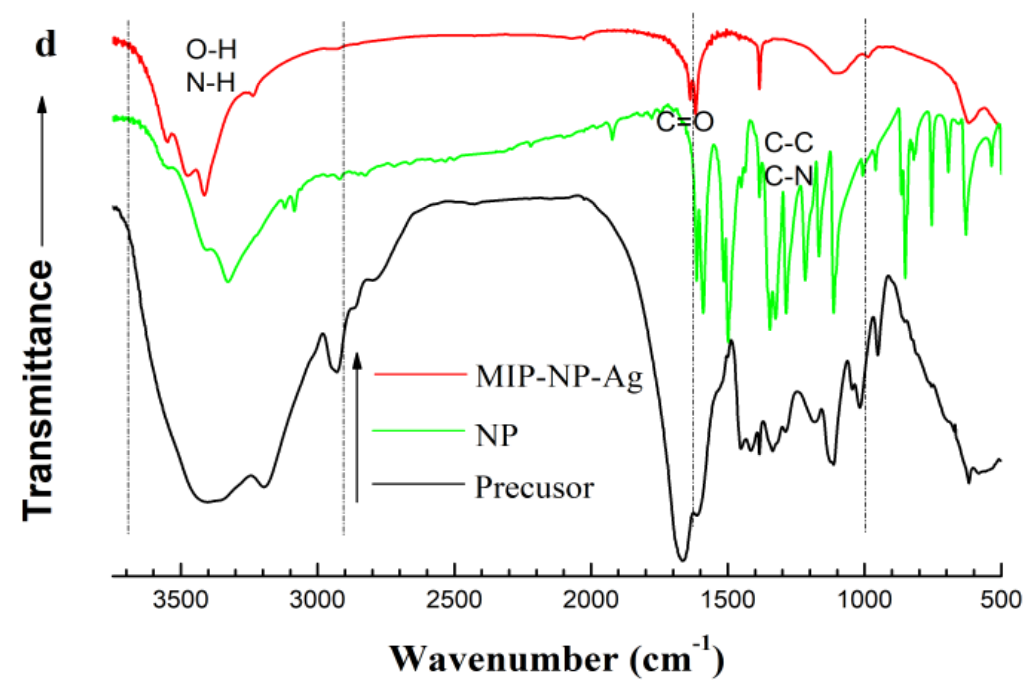

Figure 1. FTIR spectra of prepared catalysts and imprinting behavior (a: three different catalysts; b: three different layers of prepared reactor MIP-AgPRS; c: NPA imprinted layer; d: NP imprinted layer).

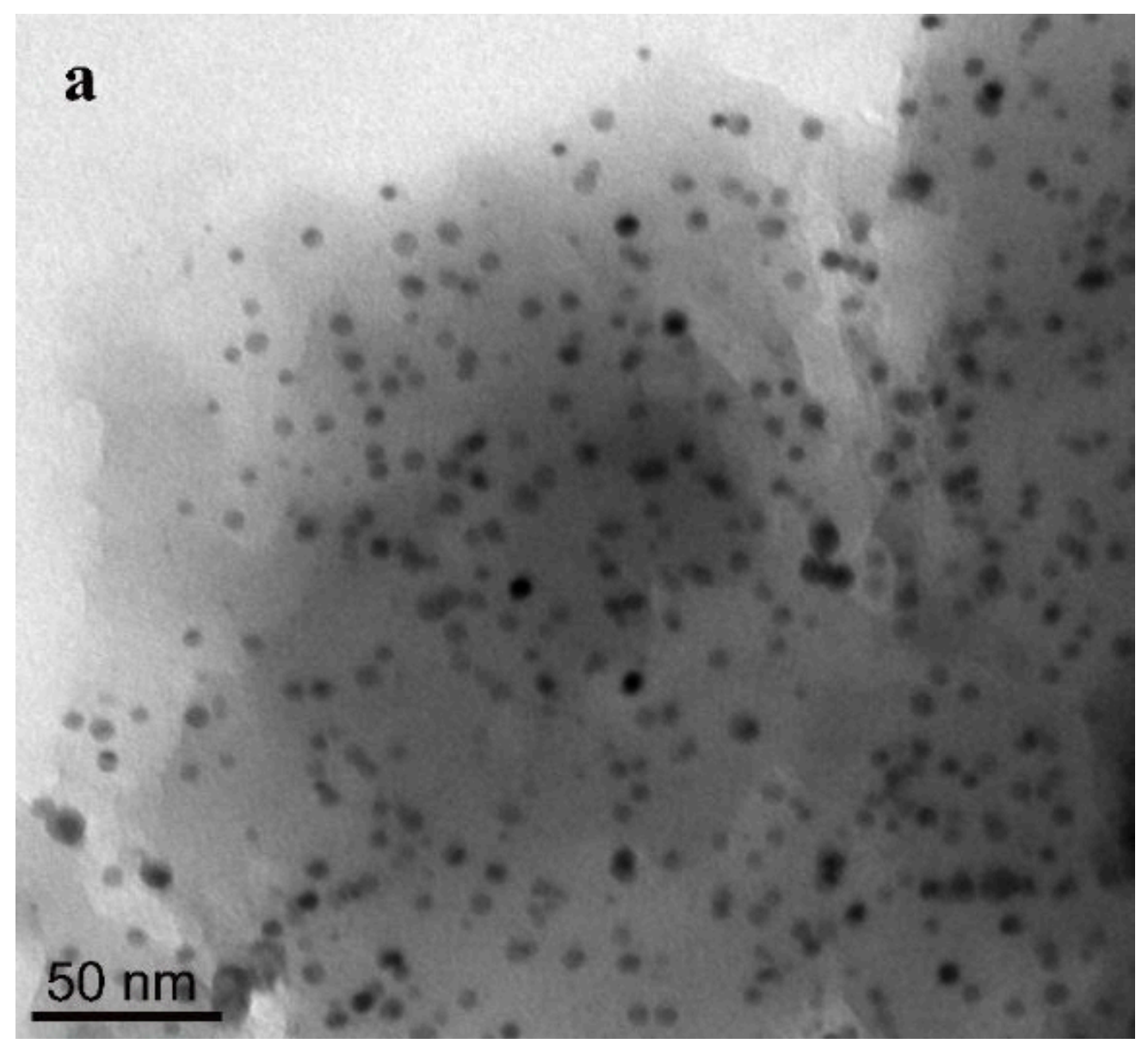




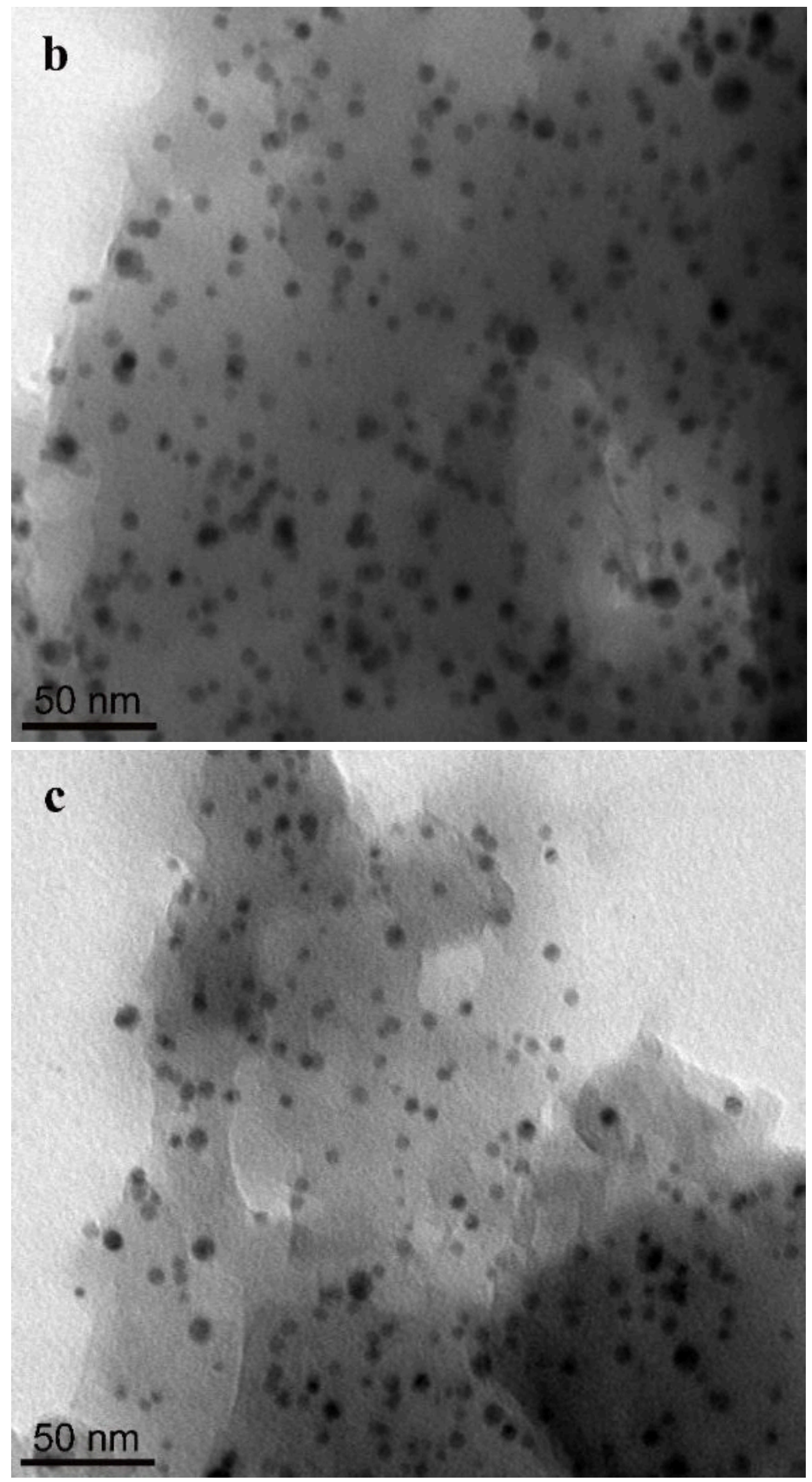


Figure 2. TEM images of metal nanoparticles in the polymer reactor and two controls. (a: MIP-AgPRS; b: MIP-AgPRO; c: MIP-AgPRC).
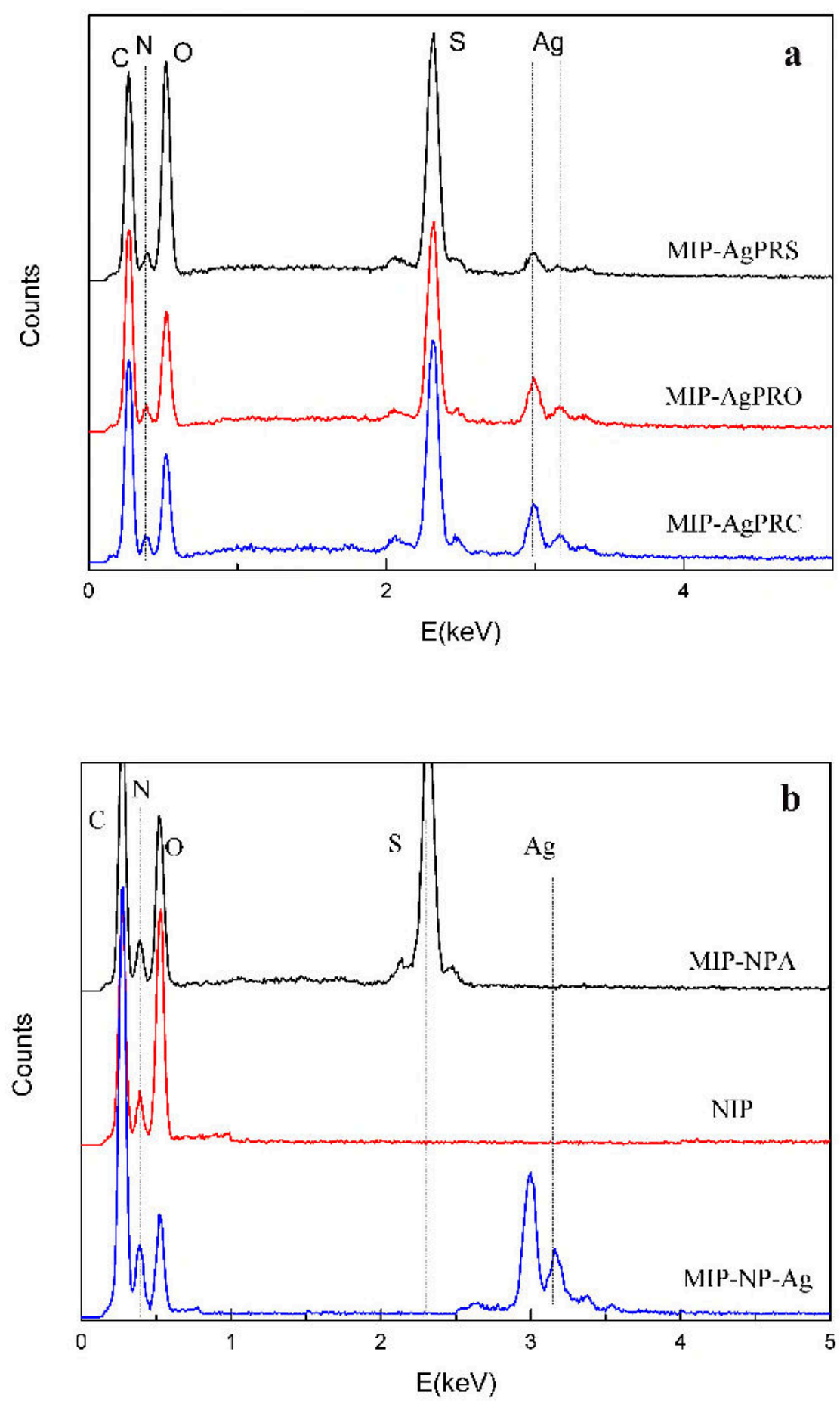

Figure 3. EDS spectra of the polymer reactors. (a: spectra of three polymer reactors; b: spectra of three layers of MIP-AgPRS). 


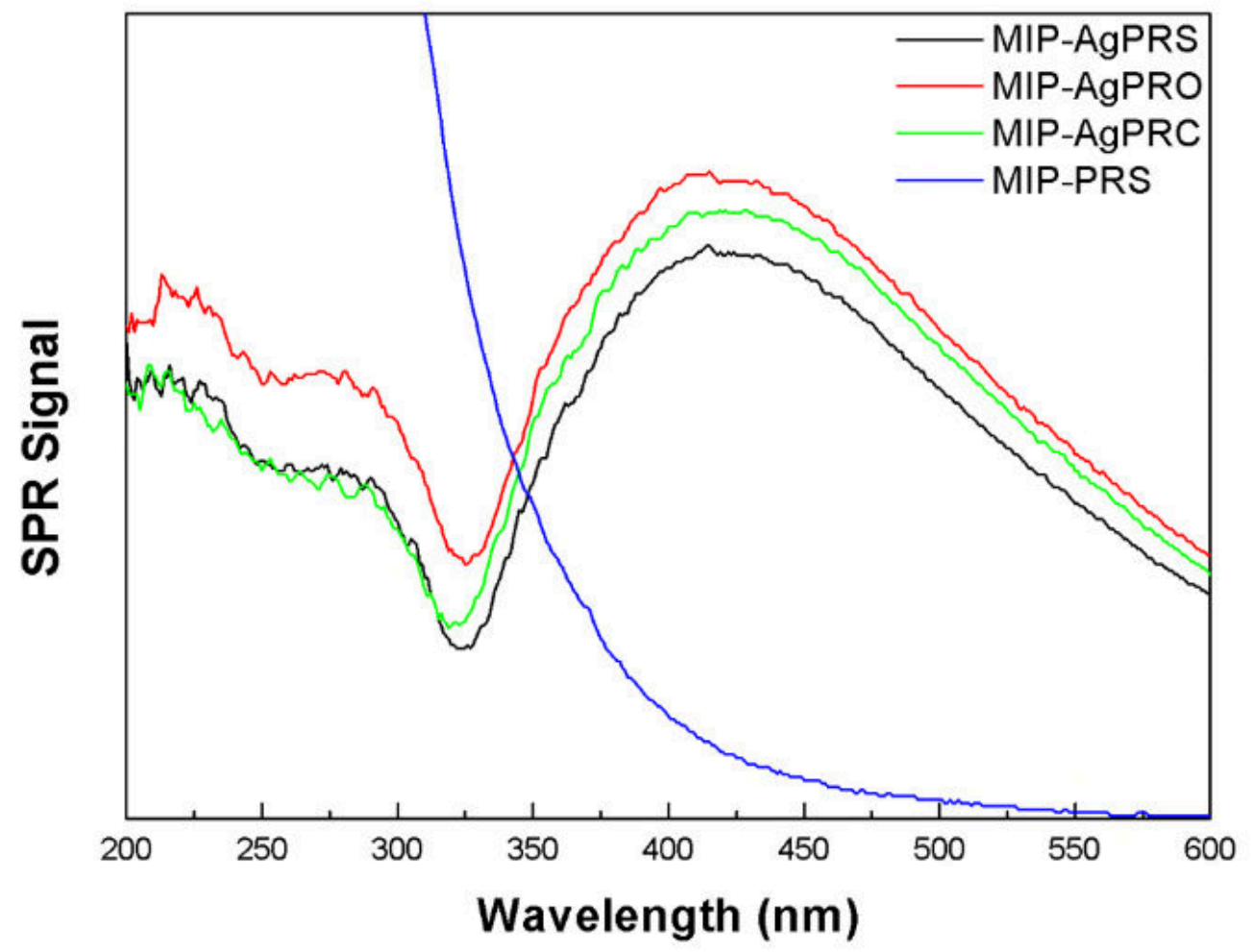

Figure 4. SPR spectra of polymer reactor and controls.

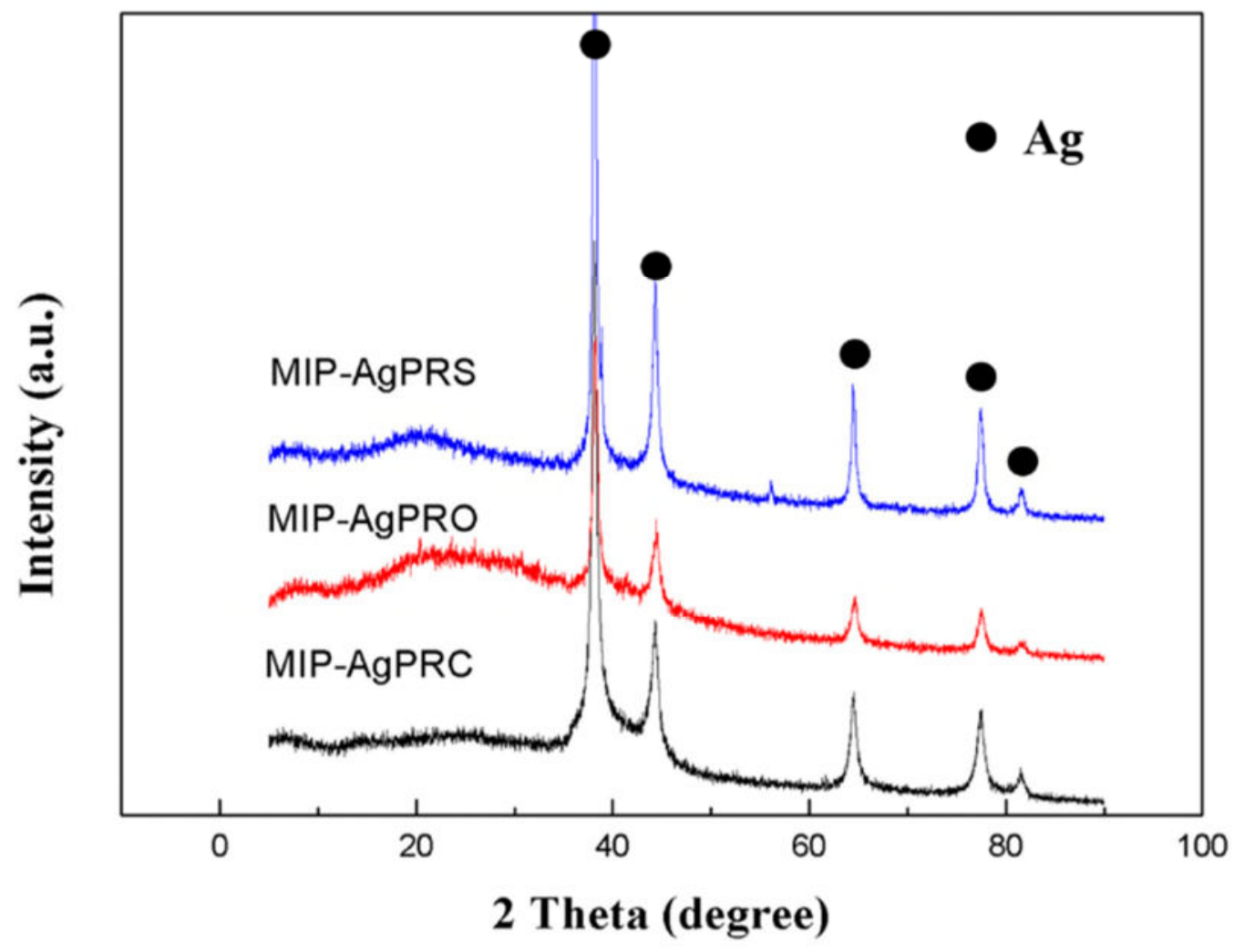


Figure 5. XRD patterns of the polymer reactor and controls.

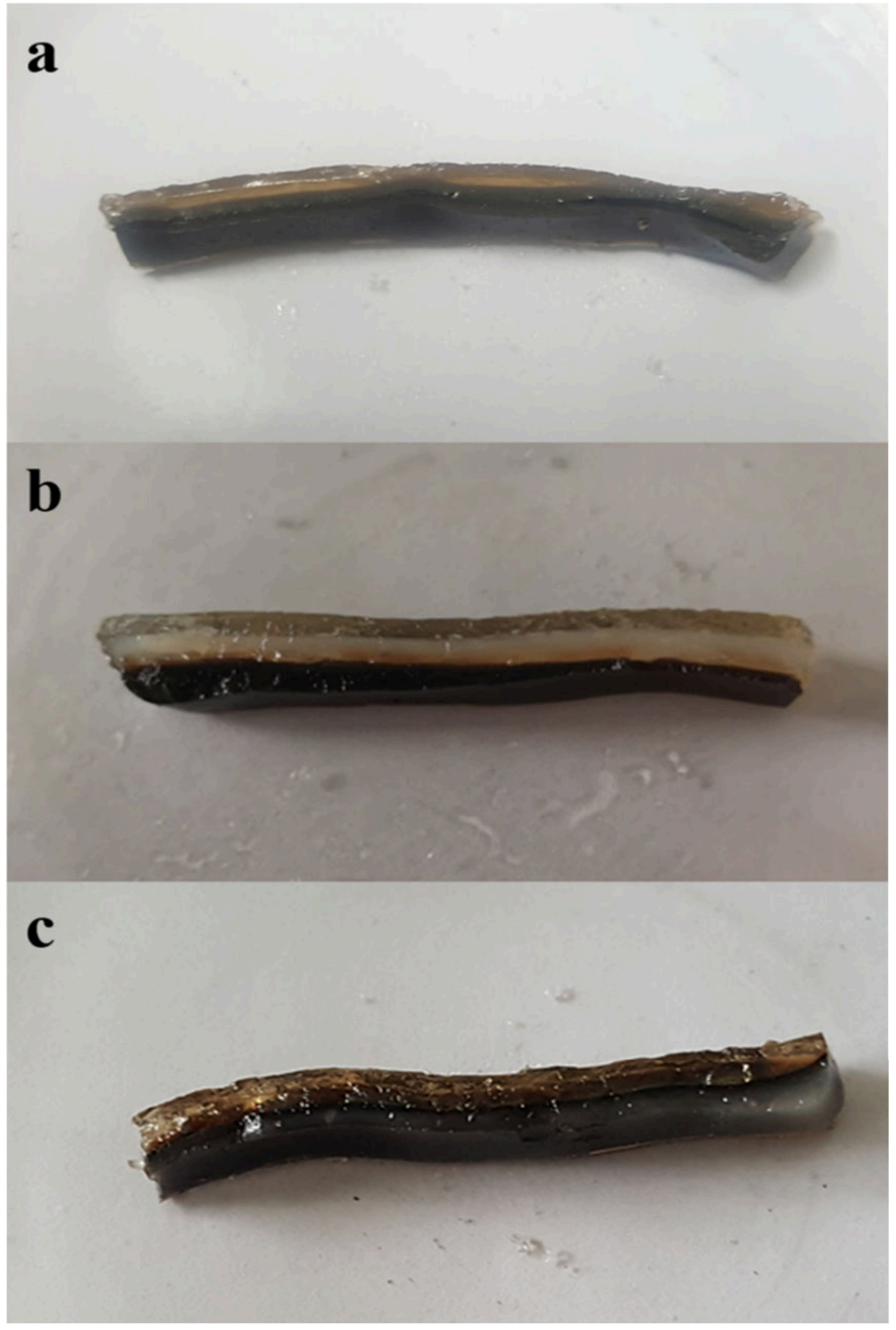

Figure 6. Digital images for a slice of the polymer reactor and two controls (a: MIP-AgPRS; b: MIP-AgPRO; c: MIP-AgPRC). 


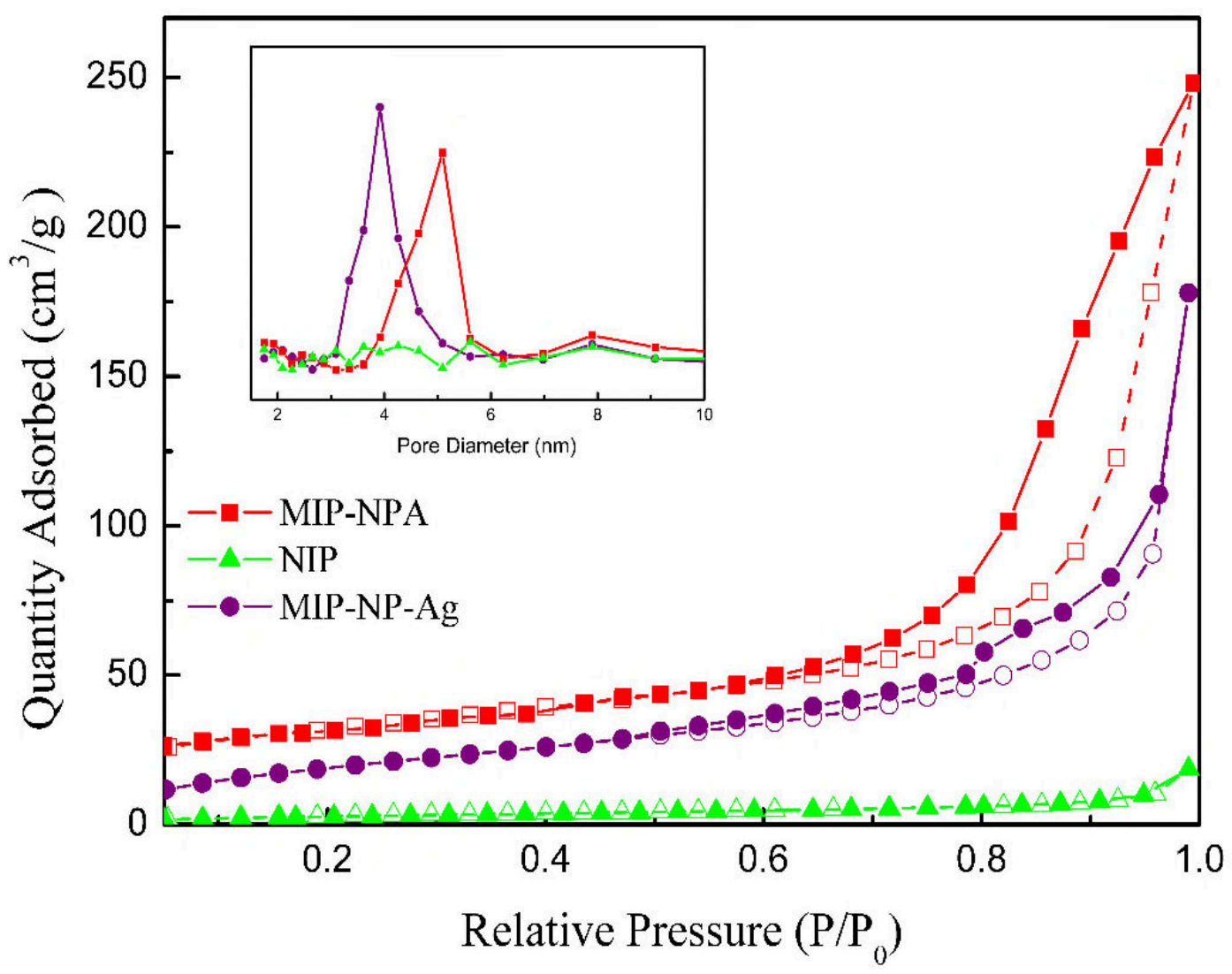

Figure 7. BET sorption isotherms of the polymer reactors MIP-AgPRS (herein, closed symbols stand for nitrogen adsorption; open symbols stand for nitrogen desorption)

Table 1. BET analysis of the different layers of polymer reactors

\begin{tabular}{|c|ccc|}
\hline & \multicolumn{3}{|c|}{ Surface area $\left(\mathbf{m}^{\mathbf{2}} \mathbf{g}^{-1}\right)$ of different samples } \\
\hline Different layer & MIP-AgPRC & MIP-AgPRO & MIP-AgPRS \\
\hline MIP-NPA & 98.7 & 112.3 & 109.5 \\
\hline NIP & 24.5 & 29.5 & 11.4 \\
\hline MIP-NP-Ag & 68.2 & 74.4 & 73.9 \\
\hline
\end{tabular}

\subsection{Temperature-programmed desorption}

The TPD tests were carried out to clarify the interaction among the different catalysts and substrates [32]. In comparison with a weaker interaction, a relatively 
strong interaction with imprinted substrate has enabled the catalysts a greater retention. Figure 8a showed the template molecule NPA desorbed from the three prepared reactors MIP-AgPRS, MIP-AgPRO, MIP-AgPRC, and the reference of the template, BNPC (bis(4-nitrophenyl) carbonate), removed from the prepared reactor MIP-AgPRS, in which the peak appeared at $321,326,326$ and $256{ }^{\circ} \mathrm{C}$, correspondingly. The imprinted substrate demonstrated stronger interactions with the prepared reactors to the non-imprinted substrate. The results were similar of the other imprinted moiety NP that NP also demonstrated stronger interactions with the three prepared reactors $(272,274$ and $273{ }^{\circ} \mathrm{C}$, respectively) (Figure 8b), compared with the non-imprinted substrate 2NP (2-Nitrophenol) desorbing from the reactor MIP-AgPRS $\left(197{ }^{\circ} \mathrm{C}\right)$. In conjunction with the results discussed in Section 4.1, it further confirmed the existence of the molecular imprinted part. As the interaction of imprinted polymers was contributed from the induced molecular memory (the imprinting process of the template molecules) [33], resulting in a more distinct interaction of the corresponding imprinted polymers.

As shown in Figure 8, the imprinted substrate NPA desorbing from all of the three imprinted reactors exhibited comparable desorption temperatures $\left(321,326\right.$ and $326^{\circ} \mathrm{C}$, respectively). The other imprinted template also showed comparable desorption temperatures in the desorbing process $\left(272,274\right.$ and $273{ }^{\circ} \mathrm{C}$, respectively). On the contrary, the non-imprinted template BNPC and 2-NP showed a relatively lower temperature (256 and $197^{\circ} \mathrm{C}$, respectively) desorbing from the corresponding imprinted reactor. The result further confirmed that the template molecules imprints were able to accommodate the respective substrate molecules. As illustrated in the preparation process (Scheme 2), the top and the bottom layer were respectively imprinted different templates, NPA and NP, allowing the two layers two different corresponding temperature. In this way, the reactors are capable of the cascade recognizable process. 


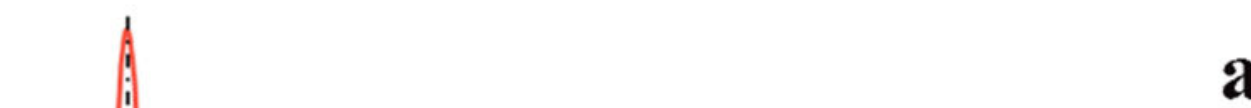

$\mathbf{a}$

Solvent

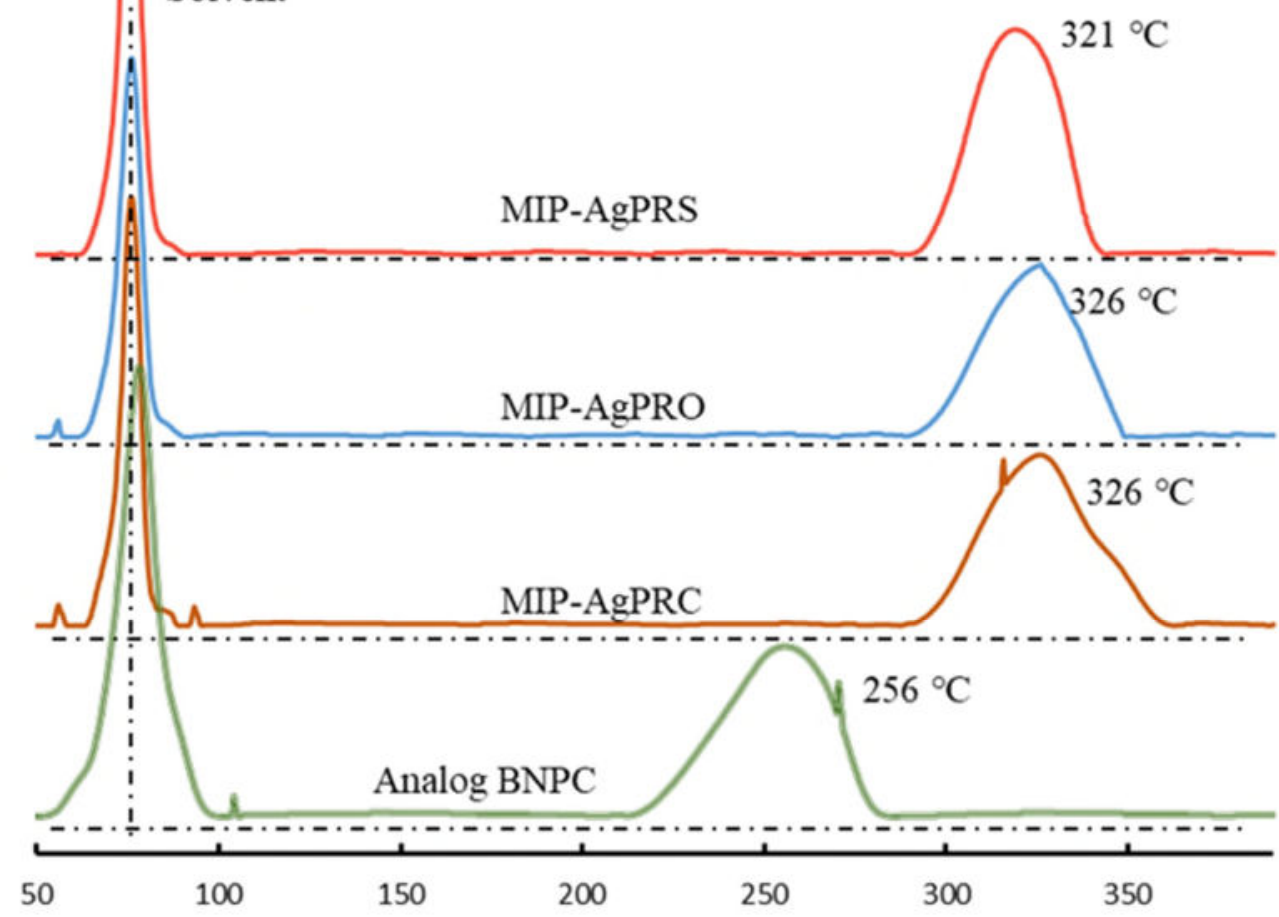

Temperature $\left({ }^{\circ} \mathrm{C}\right)$

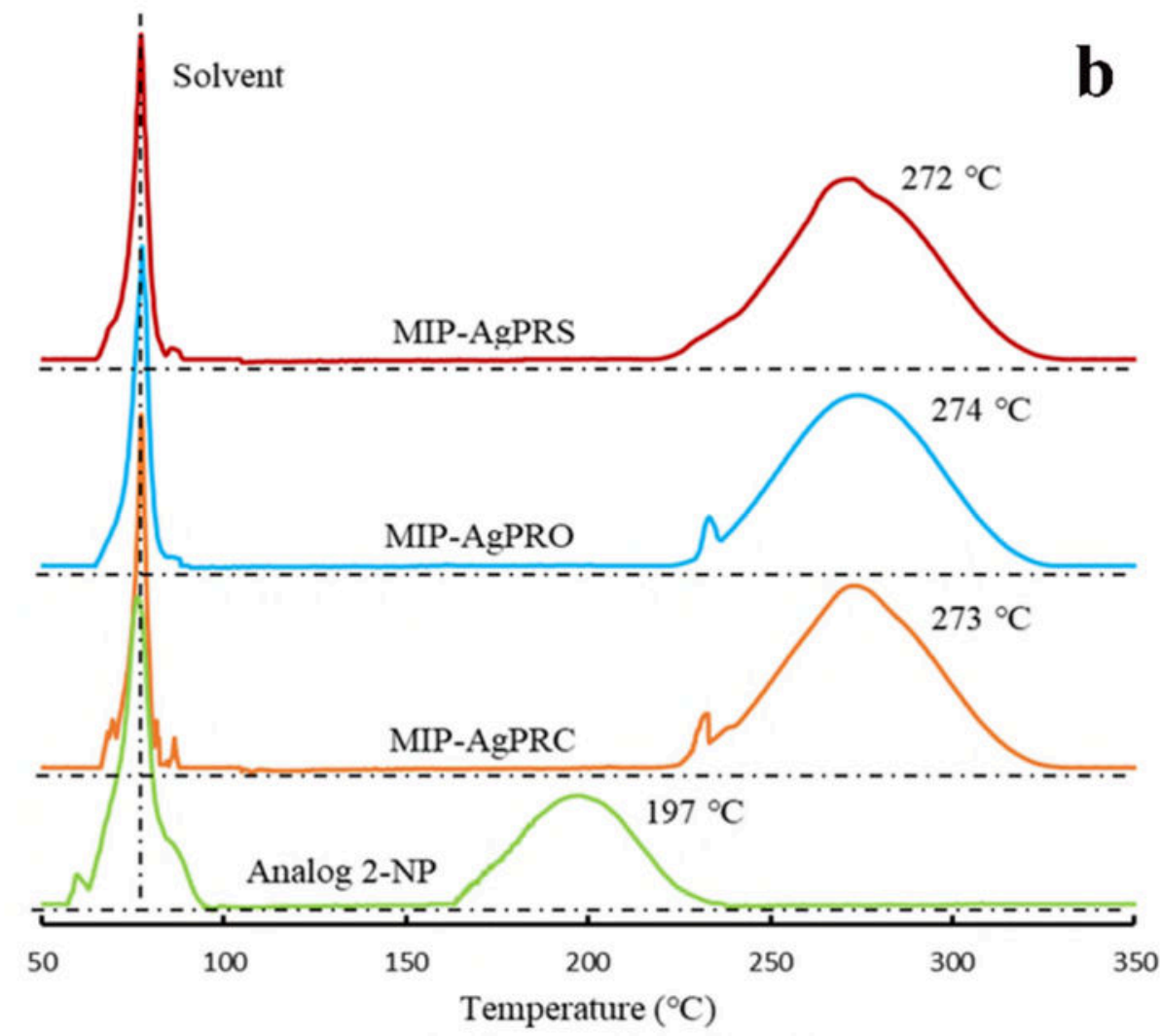

Figure 8. TPD profiles of desorbing processes to NP, NPA and reference substrate from the corresponding layer (a: NPA-imprinted layer; b: NP-imprinted layer). 


\subsection{Switchable interactions}

As shown in Figure 9a and Figure 9b, the PDPA-PAm switchable interaction was characterized according to the change of temperature. The change $\left(\mathrm{R}_{\mathrm{c}}\right)$ of swelling ratio at the switchable MIP-AgPRS demonstrated a significant dependence on temperature. However, given that the interactions in the polymeric networks of control reactors are weak and even negligible, no obvious correlation upon different temperature can be found in MIP-AgPRO and MIP-AgPRC. The dynamic radius of MIP-AgPRS amplified when the temperature rose and the dramatic surge of $\mathrm{R}_{\mathrm{c}}$ showed at $38{ }^{\circ} \mathrm{C}$. Below this temperature, MIP-AgPRS exhibited a smaller dynamic radius because of the complementary interaction between PDPA and PAm, shutting down the cascade reaction access. On the contrary, above the temperature, MIP-AgPRS showed a significant increase in the dynamic radius due to the dissociation of the PDPA-PAm interaction, resulting in open access to a tandem catalysis access. The results, therefore, demonstrated that the "on" and "off" self-healing ability of the prepared reactor was owing to the interaction between PDPA-PAm in MIP-AgPRS.

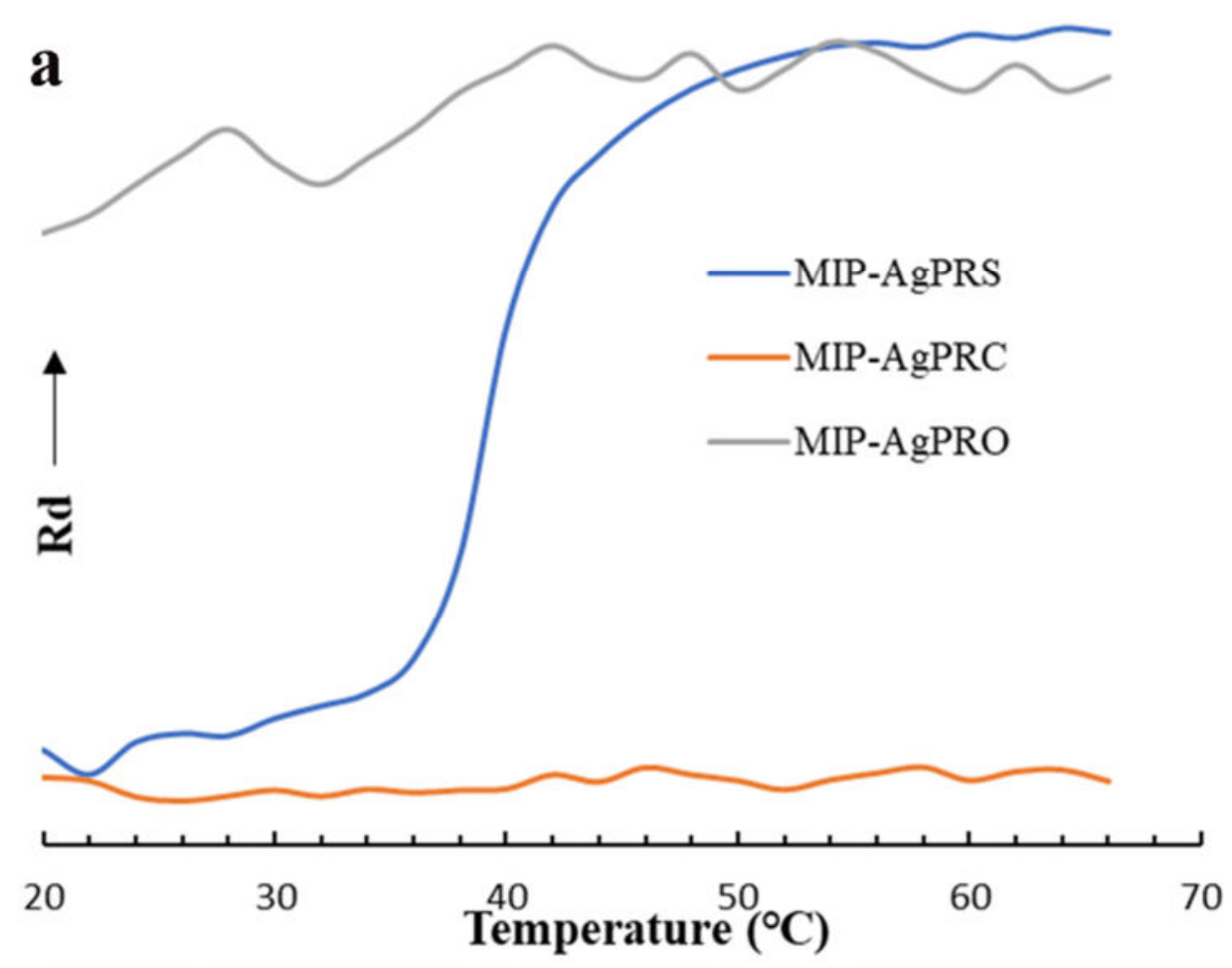




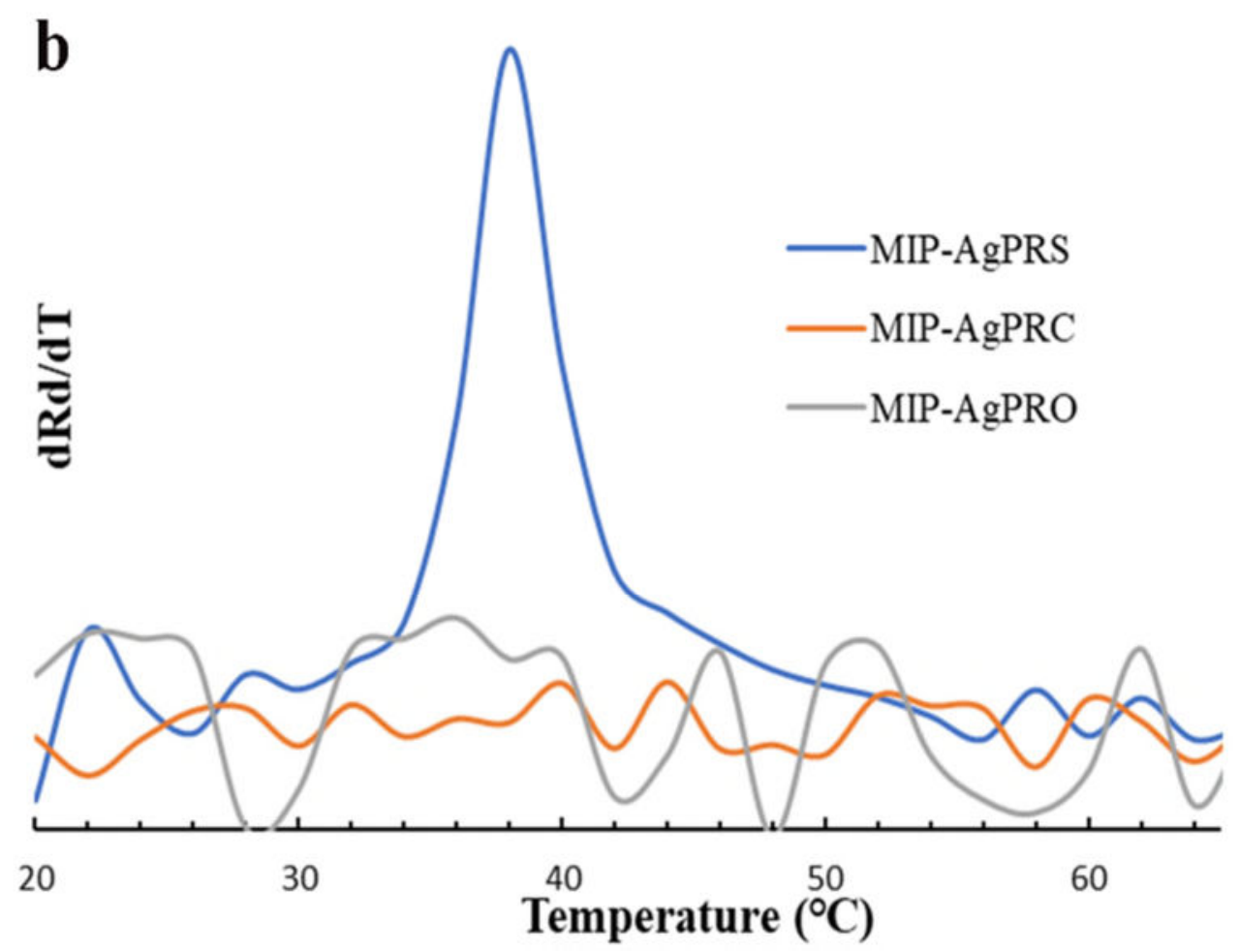

Figure 9. DLS spectra with dynamic-radius changing with the rising temperature (a: Normal; b: Differential).

\subsection{Cascade vs non-cascade catalysis with sandwiched PDPA layer switch}

To understand the catalytic process thoroughly, UV spectroscopic test was performed to record the whole reaction at both low and high temperatures. To better understand the specific position of the reactant and product in a different phase, the UV spectra of 4-nitrophenyl acetate, 4-nitrophenol and 4-aminophenol are presented firstly as reference (Figure 10).

Given that the tandem catalysis is composed of two consecutive processes, the catalytic activities were first conducted without sodium borohydride which allowed for reduction process, to get an idea about the self-hydrolysis behavior in the first step. Considering the switchable layer between the two tandem layers, two specific temperatures, 25 and $50{ }^{\circ} \mathrm{C}$ (respectively lower or higher than transition temperature $\left.\left(38^{\circ} \mathrm{C}\right)\right)$ were carefully chosen to make a comparative study. As shown in Figure 11a at $50{ }^{\circ} \mathrm{C}$, the catalytic hydrolysis of NPA at MIP-AgPRC resulted in the decrease of 
NPA $(271 \mathrm{~nm})$ and at the same time the increase of NP $(400 \mathrm{~nm})$, showing hydrolysis reaction from NPA to NP. Due to the close network in the middle layer, the tandem catalytic process was not available for this nanoreactor. However, as Figure 11c showed, the specific reactions of NPA at the reactor MIP-AgPRO was more complex, in which the decomposition of the initial substrate NPA $(271 \mathrm{~nm})$ led to the final product 4aminophenol (AP; $295 \mathrm{~nm}$ ) (Figure 11c). In this process, the intermediate NP (400 nm) hydrolyzed from NPA was then reduced by sodium borohydride to AP in the presence of the access tunnel of the middle layer. Just like the MIP-AgPRO, the switchable catalytic reactor MIP-AgPRS also demonstrated the free access to the intermediated NP, leading to the tandem catalysis at high temperature (Figure 11e).

Moreover, UV spectroscopic analysis was conducted at $25^{\circ} \mathrm{C}$ to discuss the tandem behavior at low temperatures, allowing the close networks in the middle switchable layer (which were able to prevent the intermediate to proceed the next reaction). As Figure 11d showed, the reactor MIP-AgPRO still performed the tandem reactions at the low temperature, resulting from the open networks all the time. While the prepared reactor MIP-AgPRS demonstrated only the first hydrolysis reaction (Figure 11f), the same as the MIP-AgPRC which had the closed middle layer to inhibit the access to the next reaction (Figure 11b). As we can see, the reactor MIP-AgPRC can only perform the single hydrolysis reaction while the reactor MIP-AgPRO achieves the hydrolysis to reduction the tandem process but without the control of the process. As for the switchable prepared reactor, MIP-AgPRS performed single hydrolysis at low temperature while demonstrated the tandem catalysis process at high temperature, exhibiting the self-controlled ability as expected. 

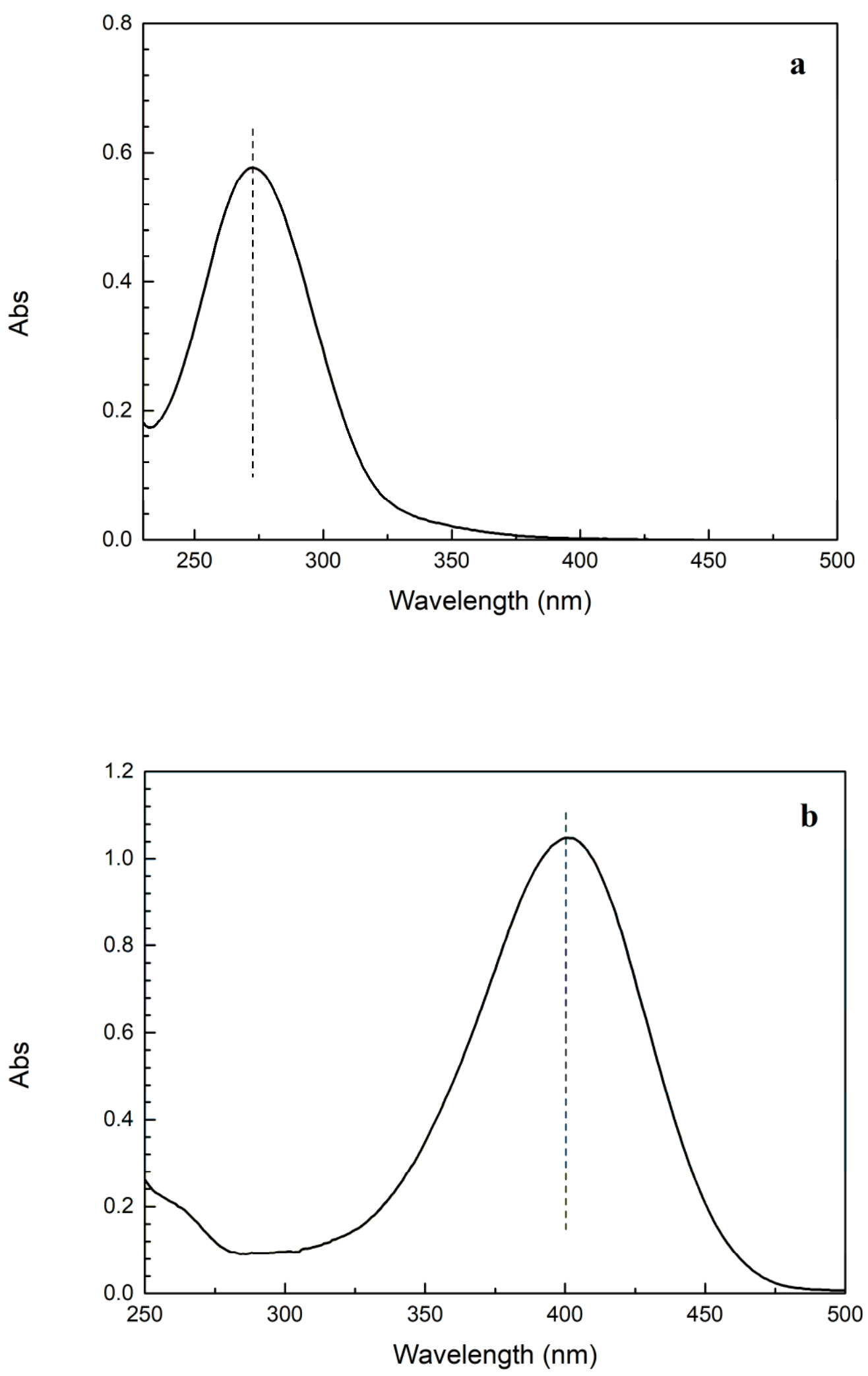


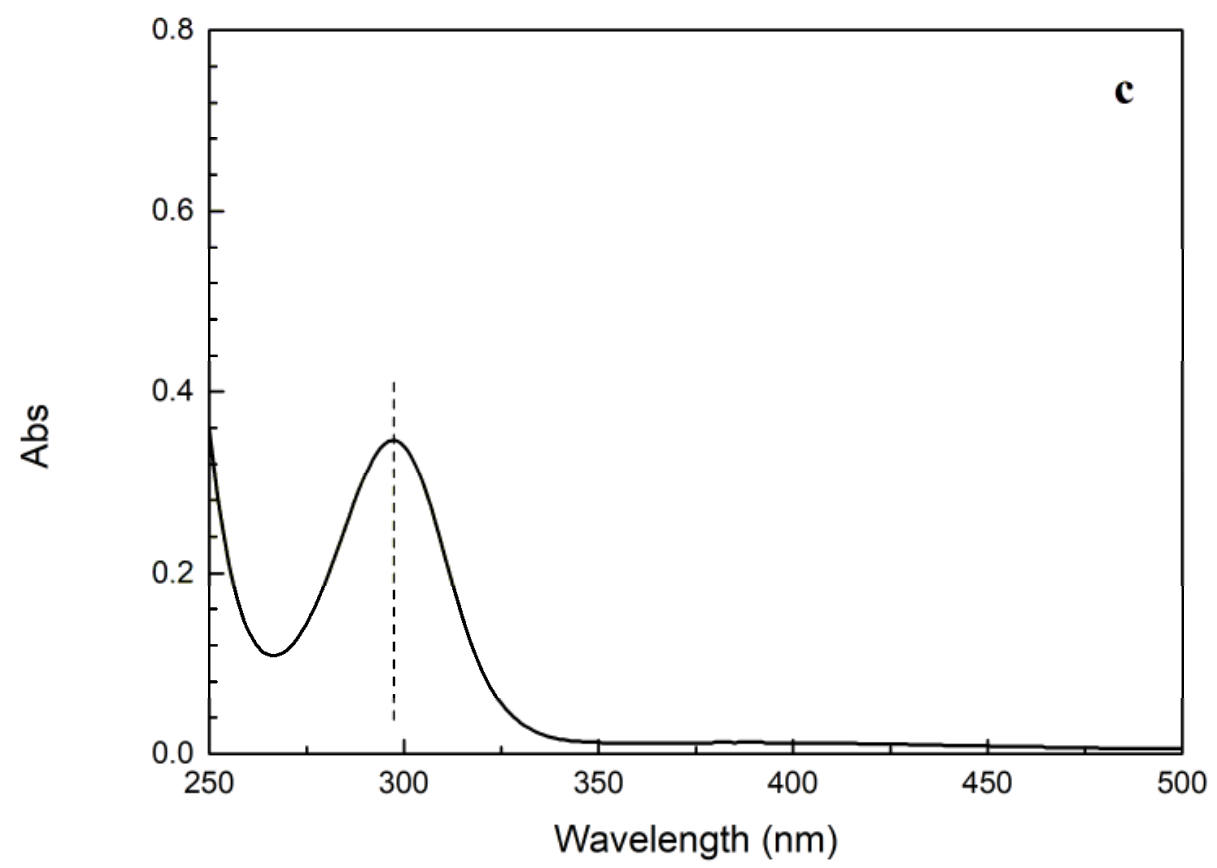

Figure 10. UV spectra of 4-nitrophenyl acetate (a), 4-nitrophenol (b) and 4aminophenol (c)

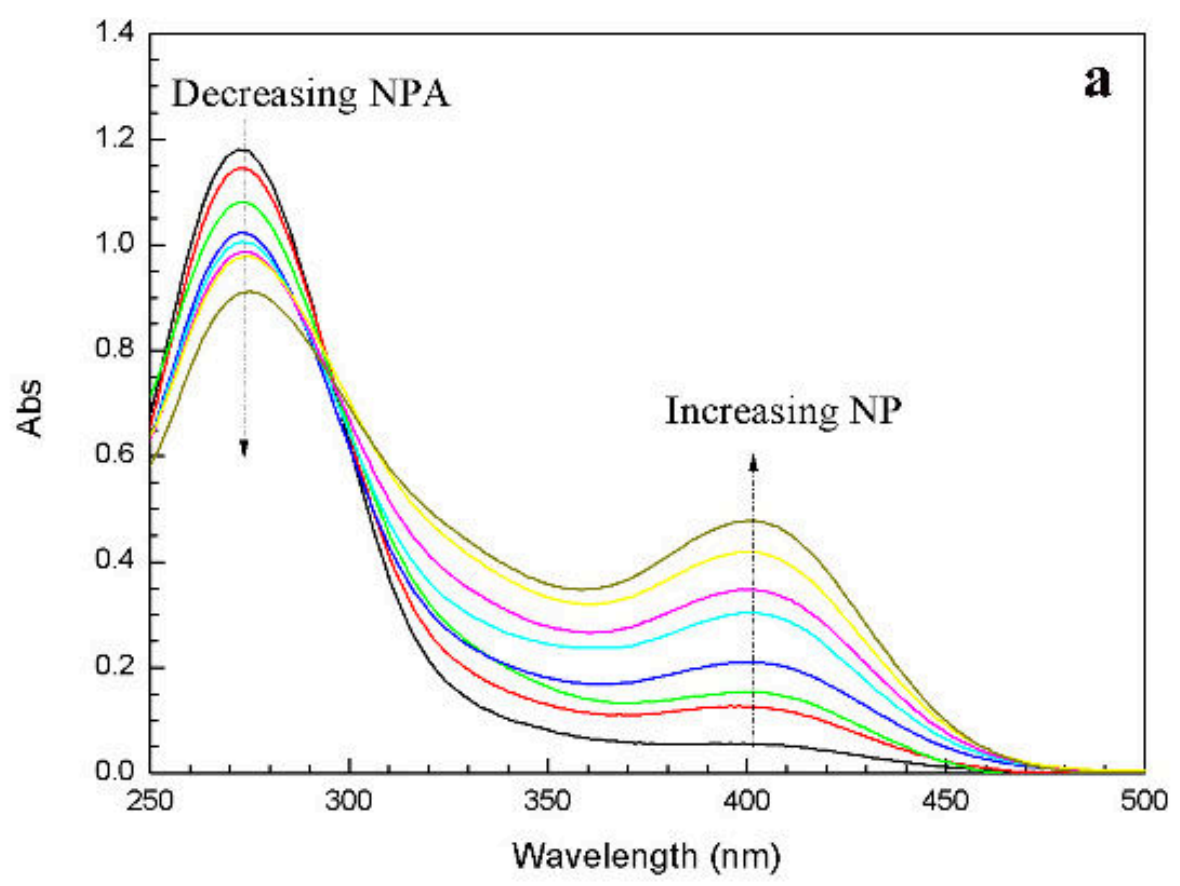



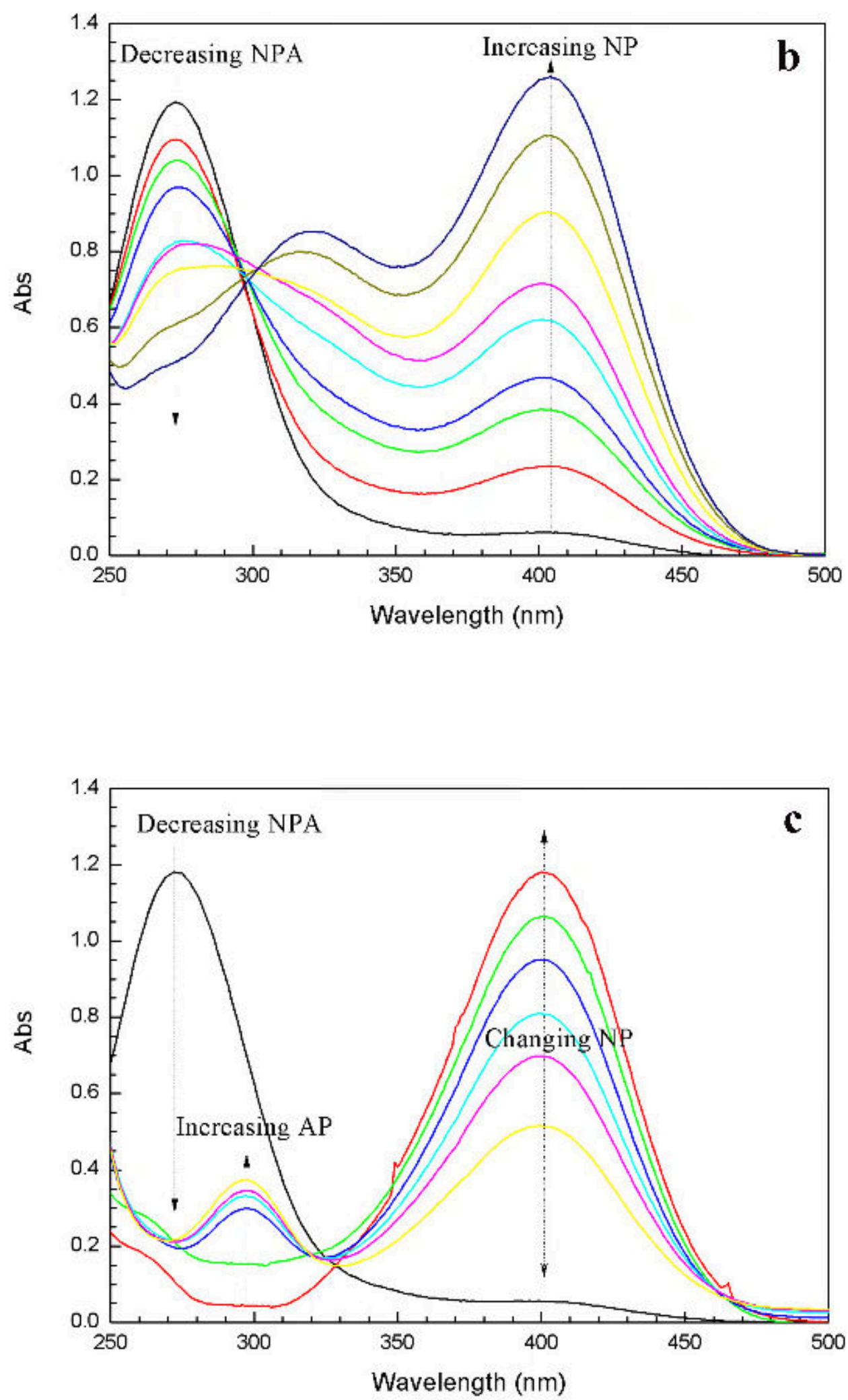

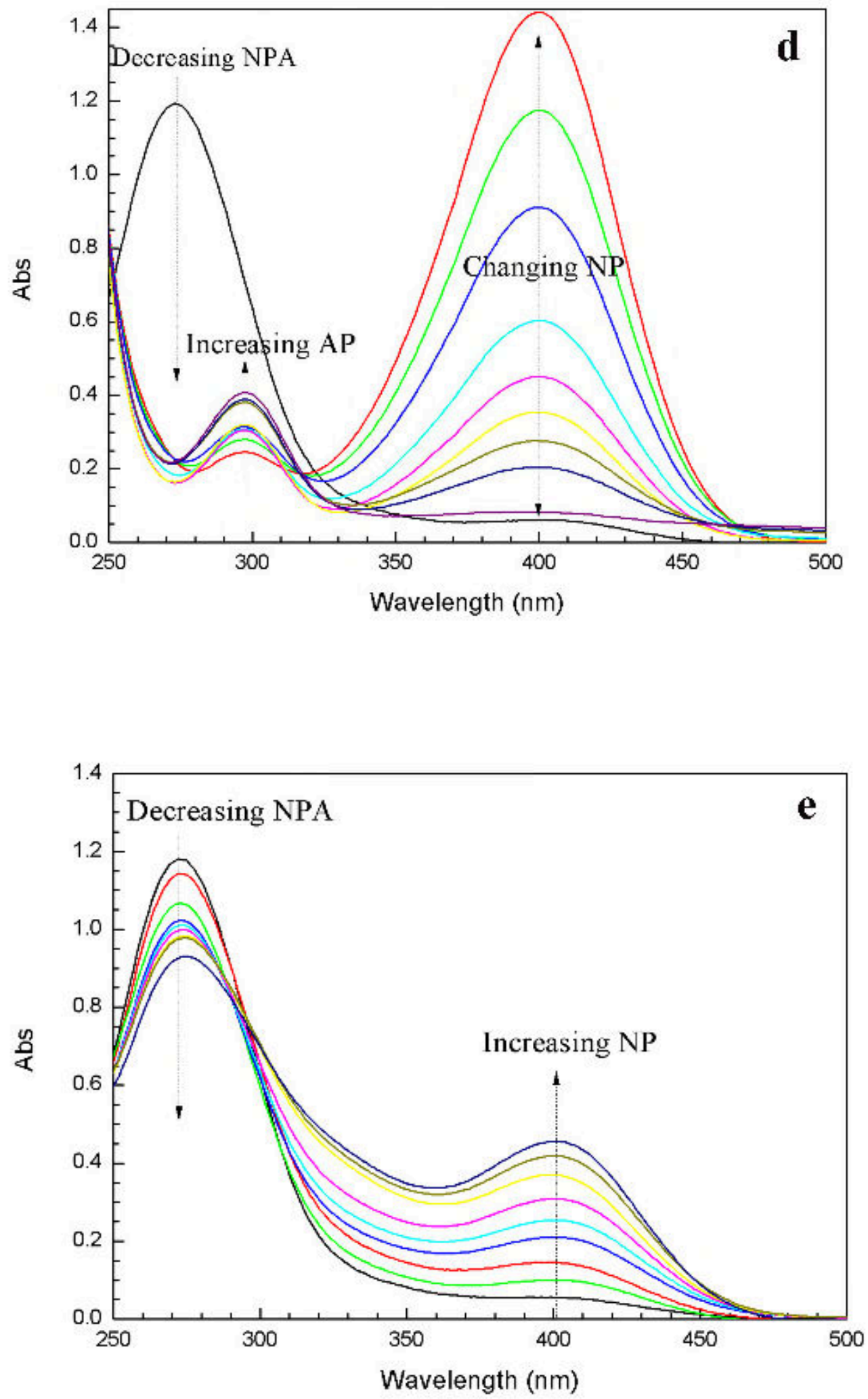


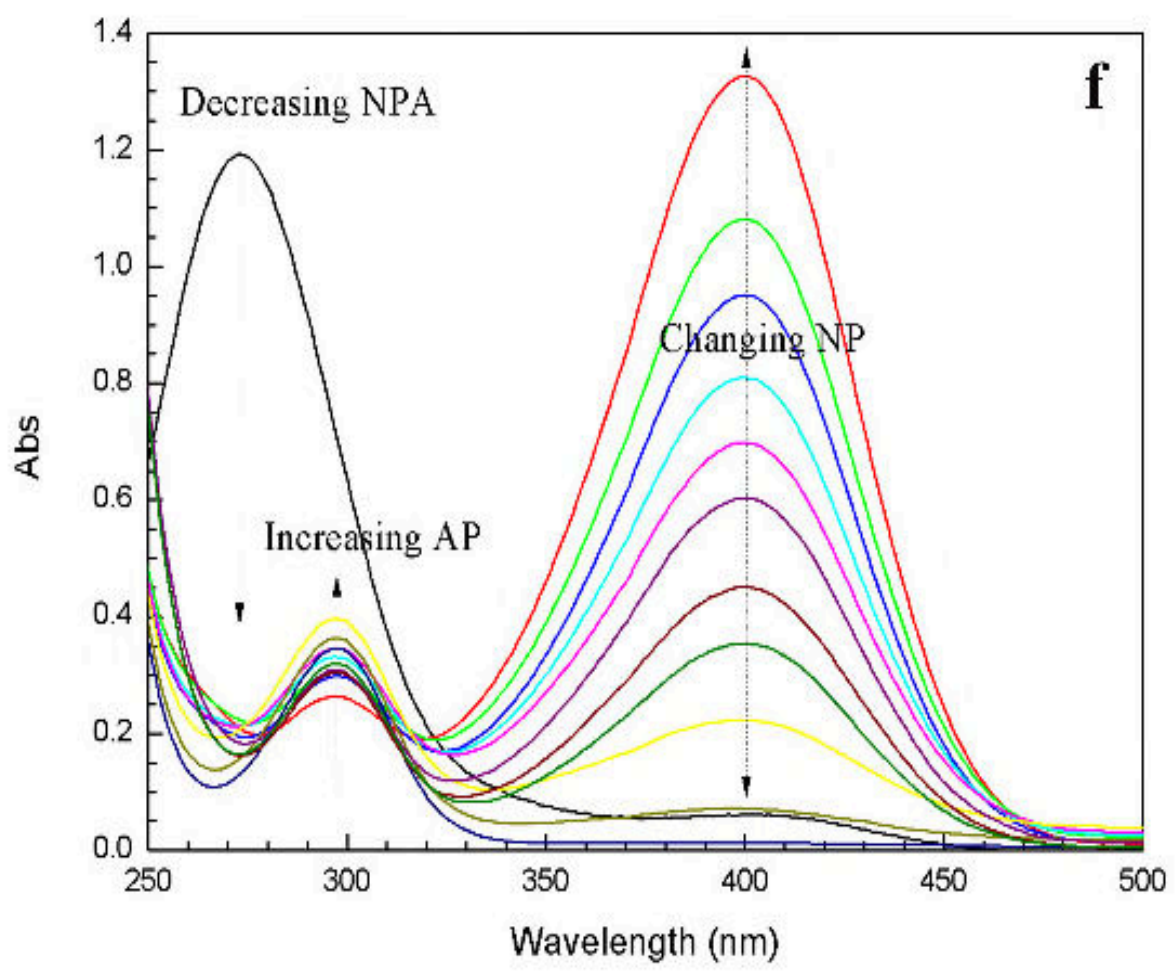

Figure 11. The changing UV spectrum of NPA at the existence of $\mathrm{NaBH}_{4}$ of the polymer reactors at different temperatures (a: MIP-AgPRC at $50^{\circ} \mathrm{C}$; b: MIP-AgPRC at $25{ }^{\circ} \mathrm{C}$; c: MIP-AgPRO at $50{ }^{\circ} \mathrm{C}$; d: MIP-AgPRO at $25{ }^{\circ} \mathrm{C}$; e: MIP-AgPRS at $50{ }^{\circ} \mathrm{C}$; $\mathrm{f}$ : MIP-AgPRS at $25^{\circ} \mathrm{C}$ ).

\subsection{Electrochemistry tests and self-healing process}

Electrochemistry (desorption) was performed to clarify the interaction among the prepared reactors as well as the substrates $[34,35]$. We know that the possibility to reduce or oxidize a molecule (binding) relies on the binding strength (among the reactors and substrates). Stronger binding would require comparatively extra energy to unbind the binding, that results in a higher redox potential. The complete mechanism (Scheme 3) have been clarified previously [36, 37]. In this reaction, substrates in the whole system commonly experience diffusion to the surface of electrodes, the desorption, and final redox processes. As the diffusion process was offset by sonication, the desorption performance of substrates closely depended on the change of the redox 
potential. In this way, the desorbing process was demonstrated inconsistent with the paradigm. Considering the switchable properties in the second PDPA-PAm layer, 25 and $50{ }^{\circ} \mathrm{C}$ were chosen again for a comparative study. As shown in Figure 12, at $25^{\circ} \mathrm{C}$, NPA attaching to the middle layers of MIP-AgPRS, MIP-AgPRO and MIP-AgPRC exhibited the reduction peaks of desorbing at $-884,-812$ and $-877 \mathrm{mV}$ respectively (a, b and c). At $50{ }^{\circ} \mathrm{C}$, the switchable nanoreactor shifted to a smaller position $(-842 \mathrm{mV})$. MIP-AgPRS exhibited a robust interaction with NPA at $30{ }^{\circ} \mathrm{C}$ than at $50{ }^{\circ} \mathrm{C}$. Connected with DLS spectra, the results suggested that the interaction offered by MIP-AgPRS showed smart control ability.

To study the interaction thoroughly, Table 2 demonstrated the reduction potentials of NPA desorbing from all the three polymer reactors. Compared with the switchable nanoreactor, the two non-responsive nanoreactors MIP-AgPRO and MIP-AgPRC did not display a significant alteration at the reduction potential of desorbing with the rising temperature. Moreover, the nanoreactor MIP-AgPRC holding close access exhibited a stronger interaction in both low and high temperatures. While the nanoreactor MIPAgPRO which always kept open access performed a weaker interaction as the temperature rose. Results strongly confirmed that the self-healing catalytic property at MIP-AgPRS existed in the second layer, allowing for the self-controlled tunnel to the cascade reactions. Therefore, the middle PDPA-PAm layer performed as a sandwiched smart switch of the access in whole tandem reactions.

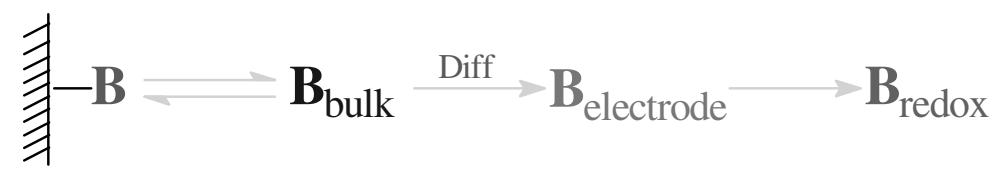

Scheme 3. A complete electrochemical process for a substrate molecule B 

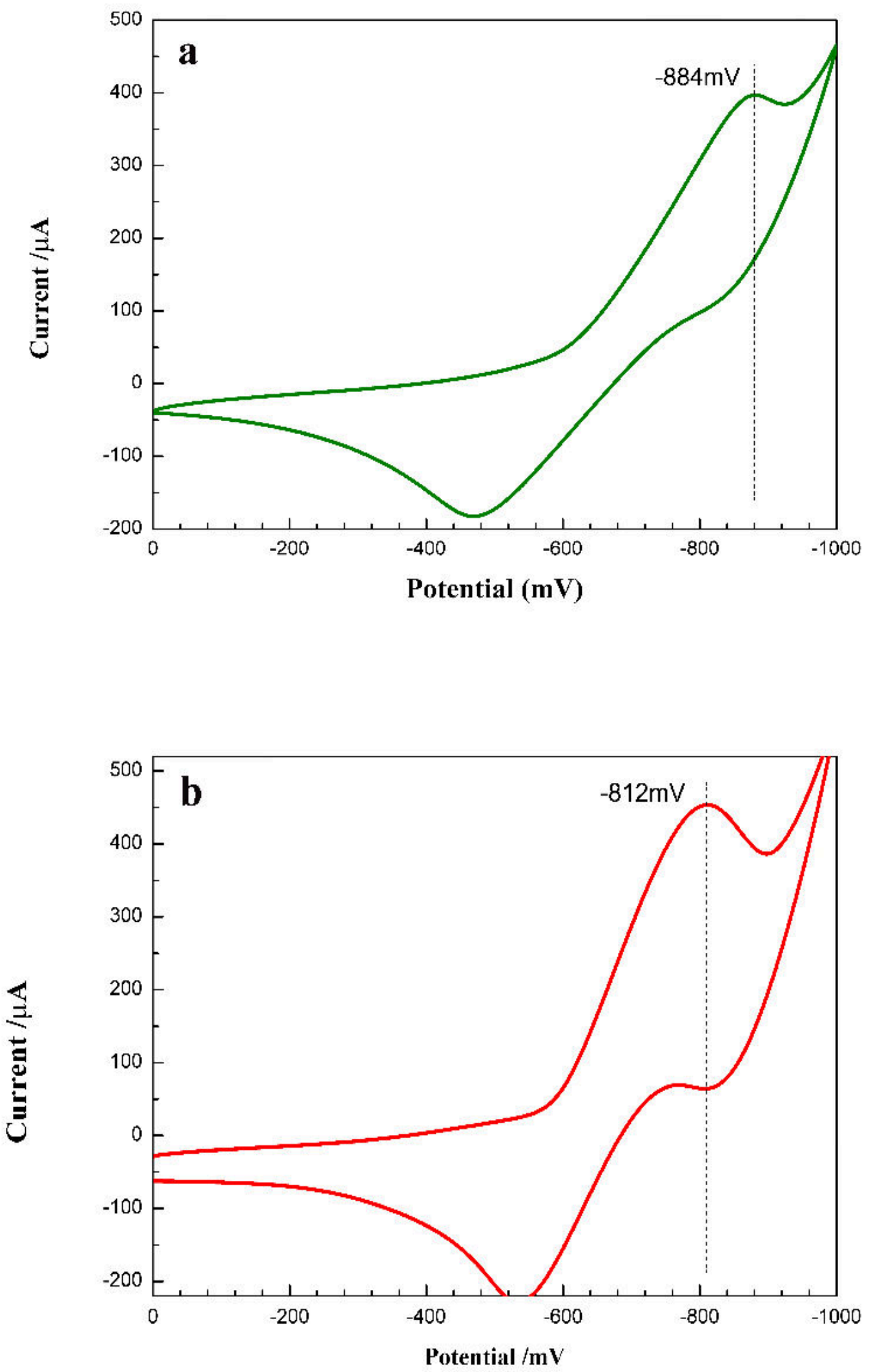

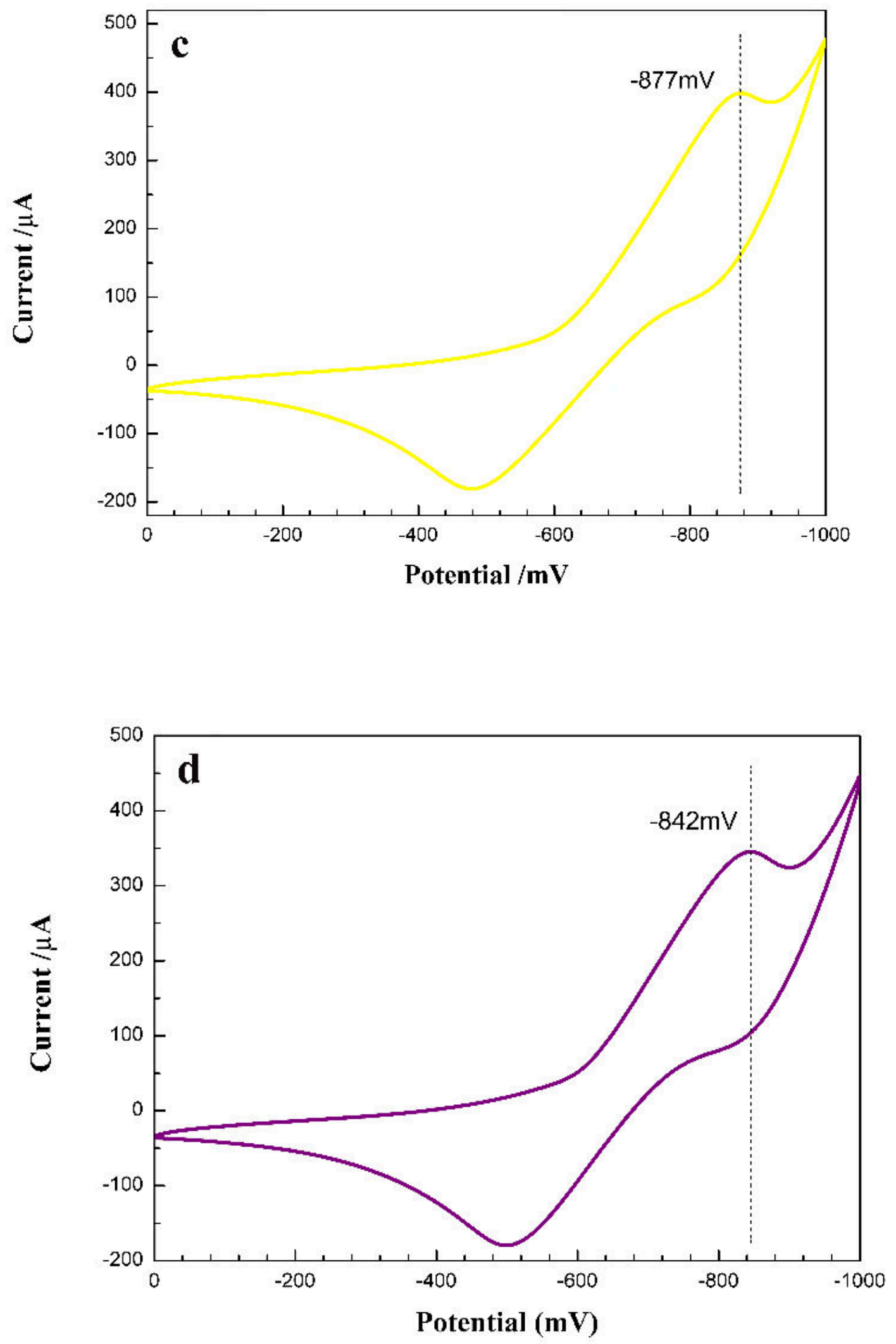

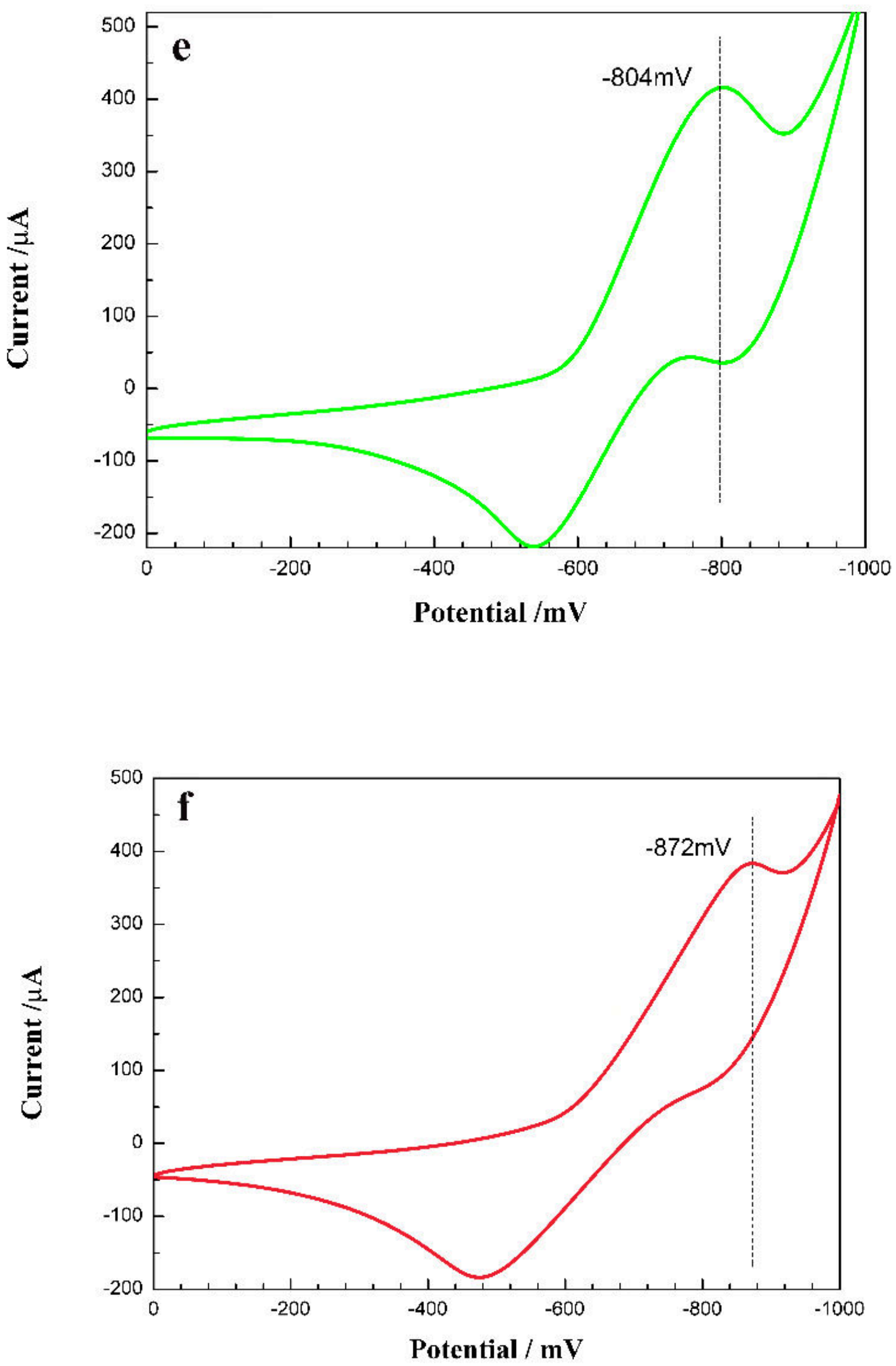
Figure 12. Reduction curves of substrates desorbing from all the polymer catalysts (a: MIP-AgPRS at $25^{\circ} \mathrm{C}$; b: MIP-AgPRO at $25^{\circ} \mathrm{C}$; c: MIP-AgPRC at $25^{\circ} \mathrm{C}$; d: MIPAgPRS at $50{ }^{\circ} \mathrm{C}$; e: MIP-AgPRO at $50{ }^{\circ} \mathrm{C}$; f: MIP-AgPRC at $50{ }^{\circ} \mathrm{C}$ ).

Table 2. Reduction potentials $(\mathrm{mV}$ ) with substrates desorbing from all the polymer catalysts.

\begin{tabular}{|c|c|c|c|}
\hline Catalytic Reactor & $25^{\circ} \mathrm{C}$ & $50^{\circ} \mathrm{C}$ & Delta \\
\hline MIP-AgPRS & -884 & -842 & 42 \\
\hline MIP-AgPRO & -812 & -804 & 8 \\
\hline MIP-AgPRC & -877 & -872 & 5 \\
\hline
\end{tabular}

\section{Conclusion}

This novel tri-layer architectural protocol is designed to solve the present problems existing in tandem catalysts. The artificial polymer reactor was composed of two molecular imprinted layers with active sites that were responsible for consecutive reactions and a sandwiched switchable layer for controlling the tandem catalytic process. The middle smart layer, prepared by mussel inspired polymer PDPA and PAm, performed like a molecular switch for the intermediate passing through to conduct the tandem catalysis. At low temperature, the switchable layer is closed, resulting in the inhibition to the intermediate. In this condition, the polymer reactor can only conduct catalytic hydrolysis. When the temperature is rising, the middle switch is open and the intermediate will pass through, enabling the complete hydrolysis to reduce tandem catalysis. Therefore, the catalytic reactor can achieve the tandem/non-tandem selfcontrolled catalysis process. The novel artificial protocol provides the potential to achieve the controllable tandem catalytic process, which may give some inspiration to the study of functional catalysts in the future.

Acknowledgements: The authors want to show gratitude to the Dual $\mathrm{PhD}$ Degree Programme between Jiangsu University and Cranfield University. Thanks also should 
be expressed to The advanced disciplinary development program in Jiangsu Province (Nos. 4013000021).

\section{Reference}

[1] C. Robert, C. M. Thomas, Tandem catalysis: a new approach to polymers, Chem. Soc. Rev. 42 (2013) 9392-9402.

[2] S. K. Raman, E. Brule, M. J. Tschan, C. M. Thomas, Tandem catalysis: a new approach to polypeptides and cyclic carbonates, Chem. Commun. 50 (2014) 1377313776.

[3] A. Corma, J. Navas, M. J. Sabater, Advances in one-pot synthesis through borrowing hydrogen catalysis, Chem. Rev. 118 (2018) 1410-1459.

[4] H. Yang, L. Fu, L. Wei, J. Liang, B. Binks, Compartmentalization of incompatible reagents within pickering emulsion droplets for one-pot cascade reactions, J. Am. Chem. Soc. 137 (2015) 1362-1371.

[5] Z. Jia, K. Wang, B. Tan, Hollow hyper-cross-linked nanospheres with acid and base sites as efficient and water-stable catalysts for one-pot tandem reactions, ACS Catal. 7 (2017) 3693-3702.

[6] H. Liu, F. Xi, W. Sun, N. Yang, E Gao, Amino- and sulfo-bifunctionalized metalorganic frameworks: one-pot tandem catalysis and the catalytic sites, Inorg. Chem. 55 (2016) 5753-5755.

[7] X. Zhang, L. Jing, F. Chang, S. Chen, H. Yang, Q. Yang, Positional immobilization of Pd nanoparticles and enzymes in hierarchical yolk-shell@shell nanoreactors for tandem catalysis, Chem. Commun. 53 (2017) 7780-7783.

[8] N. Xue, G. Zhang, X. Zhang, H. Yang, A reinforced pickering emulsion for cascade Reactions, Chem. Commun. 54 (2018) 13014-13017.

[9] H. Peng, Y. Qin, X. He, W. Li, Y. Zhang, Epitope molecularly imprinted polymer nanoparticles for chemo-/photodynamic synergistic cancer therapy guided by targeted fluorescence imaging, ACS Appl. Mater. Interfaces 12 (2020) 13360-13370.

[10] R. Gui, H. Jin, H. Guo, Z. Wang, Recent advances and future prospects in molecularly imprinted polymers-based electrochemical biosensors, Biosens. 
Bioelectron. 100 (2018) 56-70.

[11] E. Abdollahi, A. Nezhad, A. Mohammadi, M. Abdouss, M. Kalajahi, Synthesis of new molecularly imprinted polymer via reversible addition fragmentation transfer polymerization as a drug delivery system, Polymer 143 (2018) 245-257.

[12] S. Nishitani, T. Sakata, Potentiometric adsorption isotherm analysis of a molecularly imprinted polymer interface for small-biomolecule recognition, ACS Omega 3 (2018) 5382-5389.

[13] Z. Xia, W. Wei, M. Zhu, S. Wu, X. Shen. S. Li, Artificial reactor containing polymeric bilayer architectures for the formation of self-controlled tandem catalyticability, EXPRESS Poly. Lett. 14 (2020), 12-25.

[14] G. Westwood, T. N. Horton, J. J. Wilker, Simplified polymer mimics of crosslinking adhesive proteins, Macromolecules 40 (2007) 3960-3964.

[15] Y. Song, G. Ye, F. Wu, Z. Wang, S. Liu, M. Kopec, Z. Wang, J. Chen, J. Wang, K. Matyjaszewski, Bioinspired polydopamine (PDA) chemistry meets ordered mesoporous carbons (OMCs): A benign surface modification strategy for versatile functionalization, Chem. Mater. 28 (2016) 5013-5021.

[16] J. D. White, J. J. Wiker, Underwater bonding with charged polymer mimics of marine mussel adhesive proteins, Macromolecules 44 (2011) 5085-5088.

[17] W. Wei, J. Yu, C. Broomell, Hydrophobic enhancement of dopa-mediated adhesion in a mussel foot protein, J. Am. Chem. Soc. 135 (2013) 377-383.

[18] S. Hong, Y. Na, S. Choi, I. Song, W. Kim, H. Lee, Non-covalent self-assembly and covalent polymerization co-contribute to polydopamine formation, Adv. Funct. Mater. 22 (2012) 4711-4717.

[19] J. J. Wilker, Biomaterials: redox and adhesion on the rocks, Nat. Chem. Biol. 7 (2011) 579-580.

[20] Y. Liu, K. Ai, L. Lu, Polydopamine and its derivative materials: synthesis and promising applications in energy, environmental, and biomedical fields, Chem. Rev. 114 (2014) 5057-5115.

[21] B. K. Ahn, D. W. Lee, J. N. Israelachvili, Surface-initiated self-healing of polymers in aqueous media, Nat. Mater. 13 (2014) 867-872. 
[22] C. Zuo, W. Wei, Q. Zhou, S. Wu, S. Li, Artificial active nanoreactor with natureinspired sequential catalytic ability, Chemistryselect 2 (2017) 6149-6153.

[23] P. Cordier, F. Tournilhac, C. Solié-Ziakovic, L. Leibler, Self-healing and thermoreversible rubber from supramolecular assembly, Nature 451 (2008) 977-980.

[24] B. K. Ahn, D. W. Lee, J. N. Israelachvili, J. H. Waite, Surface-initiated self-healing of polymers in aqueous media, Nature Materials 13 (2014) 867-872.

[25] C. Shao, H. Chang, M. Wang, F. Xu, J. Yang, High-strength, tough, and self-healing nanocomposite physical hydrogels based on the synergistic effects of dynamic hydrogen bond and dual coordination bonds, ACS Appl. Mater. Interfaces 9 (2017) $28305-28318$.

[26] P. Xiao, S. Wu, X. Shen, M. Zhu, S. Li, Smart tandem catalyst developed with sundew's predation strategy, capable of catching, decomposing and assimilating preys, ChemCatChem 10 (2018) 5231-5241.

[27] S. Li, Y. Ge, A. Tiwari, S. Wang, A. Turner, S. Piletsky, 'On/off'-switchable catalysis by a smart enzyme-like imprinted polymer, J. Catal. 278 (2011) 173-180.

[28] Q. Chen, D. Wei, H. Chen, L. Zhu, C. Jiao, G. Liu, L. Huang, J. Yang, L. Wang, J. Zheng, Simultaneous enhancement of stiffness and toughness in hybrid double-network hydrogels via the First, physically linked network, Macromolecules 48 (2015) 80038010.

[29] C. Wang, J. Bai, Y. Liu, X. Jia, X. Jiang, Polydopamine coated selenide molybdenum: A new photothermal nanocarrier for highly effective chemophotothermal synergistic therapy, ACS Biomater. Sci. Eng. 2 (2016) 2011-2017.

[30] S. Link, M. A. Elsayed, Spectral properties and relaxation dynamics of surface plasmon electronic oscillations in gold and silver nanodots and nanorods, J. Phys. Chem. B. 103 (1999) 8410-8426.

[31] A. Pan, Z. Yang, H. Zheng, F. Liu, Y. Zhu, X. Su, Z. Ding, Changeable position of SPR peak of $\mathrm{Ag}$ nanoparticles embedded in mesoporous $\mathrm{SiO}_{2}$ glass by annealing treatment, Applied Surface Science 205 (2003) 323-328.

[32] W. Wei, T. Zhou, S. Wu, X. Shen, M. Zhu, S. Li, An enzyme-like imprintedpolymer reactor with segregated quantum confinements for a tandem catalyst, RSC Adv. 
8 (2018) 1610-1620.

[33] J. Liu, H. Song, J. Liu, Y. Liu, L. Li, H. Tang, Y. Li, Preparation of molecularly imprinted polymer with double templates for rapid simultaneous determination of melamine and dicyandiamide in dairy products, Talanta 134 (2015) 761-767.

[34] Y. Han, X. Yuan, M. Zhu, S. Li, M. Whitcombe, S. Piletsky, A catalytic and shapememory polymer reactor, Adv. Funct. Mater. 24 (2014) 4996-5001.

[35] R. Luo, M. Zhu, X. Shen, S. Li, Polymer catalyst with self-assembled hierarchical access for sortable catalysis, J. Catal. 331 (2015) 49-56.

[36] X. Zheng, R. Luo, M. Zhu, S. Li, Polymer nanoreactor with "mobility-recalling" domains for on/off switchable catalysis, ChemCatChem 7 (2015) 814-818.

[37] J. Wang, M. Zhu, X. Shen, S. Li, A cascade-reaction nanoreactor composed of a bifunctional molecularly imprinted polymer that contains Pt nanoparticles, Chem. Eur. J. 21 (2015) 7532-7539. 
2020-05-18

\section{pÿTowards next generation smart tandem catalysts with sandwiched mussel-inspired layer switch}

Wei, Wenjing

Elsevier

pÿWei W, Thakur VK, Chew JYM, Li S. (2020) Towards next generation smart tandem catalysts with sandwiched mussel-inspired layer switch. Materials Today Chemistry, Volume 17, September 2020, Article number 100286 https://doi.org/10.1016/j.mtchem.2020.100286

Downloaded from Cranfield Library Services E-Repository 